\title{
STRONG SHAPE FOR TOPOLOGICAL SPACES
}

\author{
JERZY DYDAK AND SLAWOMIR NOWAK
}

\begin{abstract}
Strong shape equivalences for topological spaces are introduced in a way which generalizes easily to inverse systems of topological spaces. Each space is then mapped via a strong shape equivalence into a fibrant inverse system of ANRs. This leads naturally to defining the strong shape category SSh for topological spaces. Other descriptions of SSh are also provided.
\end{abstract}

\section{INTRODUCTION}

Strong shape category for topological spaces was introduced by D. A. Edwards and $\mathrm{H}$. Hastings in their excellent monograph [E-H]. They introduce Steenrod homotopy category ho(pro-TOP) for pro-spaces which is a model category in the sense of Quillen. Then one has to associate an appropriate inverse system $X^{\prime}$ of ANRs with a given space $X$ and define strong shape morphisms from $X$ to $Y$ as morphisms of ho(pro-TOP) from $X^{\prime}$ to $Y^{\prime}$. Edwards and Hastings chose the Vietoris system $V(X)$ as $X^{\prime}$. The drawback of that is that there is no morphism from $X$ to $V(X)$ in pro-TOP. This was remedied by Cathey and Segal [C-S] who chose Mardešić resolution $M(X)$ of $X$ as $X^{\prime}$. However, Mardešić resolution is not a functor from TOP to pro-TOP. Such a functor exists in the compact metrizable case (see [C-H]).

For some the approach in [E-H] seems too abstract, so there was a need to introduce strong shape category in a more geometric way. Such a task was undertaken by Lisica and Mardešić [L-M $\left.{ }_{1}\right]$. However, they ran into many technical difficulties; some of the notions are hard to visualize and their geometric intuition gets lost. In this paper we produce for each cardinal number a new kind of resolution which is a functor from TOP to pro-TOP. The unifying idea is that of a strong shape equivalence. A map $f: X \rightarrow Y$ is a strong shape equivalence if both $f$ and the natural projection $p: D M(f) \rightarrow Y$ from the double mapping cylinder of $f$ to $Y$, are shape equivalences. This allows us to use (in the paracompact case) the double mapping cylinder trick of Kozlowski $[\mathrm{K}]$. The reason it is so useful is because it is the geometric equivalent of the Mayer-Vietoris exact sequence for cohomology.

Received by the editors June 30, 1988 and, in revised form, February 7, 1989.

1980 Mathematics Subject Classification (1985 Revision). Primary 55P55; Secondary 54C55.

Key words and phrases. Shape, fibrations, homotopy equivalences, absolute neighborhood retracts, function spaces. 
In the first part we prove general results concerning strong shape equivalences. Some of them are generalizations of the corresponding results in the compact metrizable case (see [D-S, $\mathrm{C}$ and $\mathrm{C}-\mathrm{H}]$ ) and some are new (Theorem 1.8). $\S 2$ is devoted to the paracompact case. Theorem 2.7 is a variation of the main result of $[\mathrm{K}]$; we think that our proof is quite simple and is a good example of how the double mapping cylinder works. $\S 3$ is devoted to fibrant diagrams. We changed the original definition from [E-H] to a more symmetrical one. This allows us to prove the main result (Theorem 3.5) quite easily. Notice that no advanced ANR theory is used in this paper (in contrast to [L-M $\left.\mathrm{M}_{1}\right]$ ); Theorem 3.5 is all we need. Our definition of strong shape equivalences was chosen so that it generalizes without any changes to pro-TOP $(\S 4)$. Now, the basic idea is to find a resolution $X^{\prime}$ of a topological space $X$ such that (a) $X \rightarrow X^{\prime}$ is a strong equivalence and (b) $X^{\prime}$ is fibrant. This is done in Theorem 4.6 and the construction is quite simple. We obtain a characterization of strong shape equivalences similar to that of shape equivalences (Theorem 4.7). Also we give a description of the strong shape category SSh in a way resembling Mardešić definition of shape category (Theorem 5.9). $\S 5$ contains a result (Theorem 5.6), previously overlooked, which establishes a connection between Ferry's $\left[F_{1}\right]$ question of improving compacta and that of isomorphism between SSh and the category obtained from HTOP by formally inverting strong shape equivalences. If $X$ is a closed subset of an ANR-space $M$, then the system of neighborhoods of $X$ in $M$ provides a natural resolution $X^{\prime}$ such that $X \rightarrow X^{\prime}$ is a strong shape equivalence. The problem is that $X^{\prime}$ is not fibrant. Using $\S 3$ we provide a simple description (in $\S 6$ ) of a fibrant system which is equivalent to $X^{\prime}$. This allows us to describe morphism of SSh to $X$ in a way analogous to Quigley's [Q] approaching maps. By switching from homotopy to homology one arises naturally at strong $k$-homology groups ( $k$ is any integer) which are isomorphic to Lisica-Mardešic [L-M ${ }_{2}$ ] strong homology groups (see [M-P] for their definition). Also we apply our results to conclude that the function spaces $P^{X}$ and $P^{Y}$ are homeomorphic if $X$ and $Y$ are nondegenerate compacta of the same shape and $P$ is separable and completely metrizable ANR with no isolated points. This result is a form of duality. It is well known that homology of $\left(S^{n}\right)^{X}$ is closely related to cohomology of $X$ (see [Mo]).

The authors are grateful to Sibe Mardešic for many valuable comments and improvements of the paper.

\section{StRong Shape EQUivalenCES BETWEen topological SPACES}

Recall that a map $f: X \rightarrow Y$ is a shape equivalence if for each $P \in$ ANR (here ANR means ANR for metric spaces), the function $f^{*}:[Y, P] \rightarrow[X, P]$ is a bijection between sets of homotopy classes $[Y, P]$ and $[X, P]$. Thus $g f \approx h f, g, h: Y \rightarrow P$, implies $g \approx h$. Strong shape equivalences are maps for which a stronger property holds: 
1.1. Definition. A shape equivalence $f$ is called a strong shape equivalence if for any maps $g, h: Y \rightarrow P \in$ ANR and a homotopy $H: H \times I \rightarrow P$ joining $g f$ and $h f, H$ is homotopic rel. $X \times\{0,1\}$ to $H^{\prime} \cdot(f \times$ id $)$ for some homotopy $H^{\prime}: Y \times I \rightarrow P$ joining $g$ and $h$.

The homotopy $H^{\prime}$ in the above definition is unique in the sense that any other homotopy $H^{\prime \prime}: Y \times I \rightarrow P$ joining $g$ and $h$ such that $H$ is homotopic rel. $X \times\{0,1\}$ to $H^{\prime \prime} \cdot(f \times$ id $)$ must be homotopic to $H^{\prime}$ rel. $Y \times\{0,1\}$. More generally, the following holds $(n=2$ corresponds to the above statement).

1.2. Proposition. If $f: X \rightarrow Y$ is a shape equivalence and $g: \partial I^{n} \times Y \rightarrow P \in$ ANR is a map such that $g(\mathrm{id} \times f)$ extends onto $I^{n} \times X$, then $g$ extends onto $I^{n} \times Y$.

Proof. $g$ can be thought of as a map $g: Y \rightarrow P^{\partial I^{n}}$. Let $h: X \rightarrow P^{I^{n}}$ be an extension of $g f$. Since $f$ is a shape equivalence, there is a map $h^{\prime}: Y \rightarrow P^{I^{n}}$ with $h^{\prime} f \approx h$. Now, treat $h^{\prime}$ as $h^{\prime}: I^{n} \times Y \rightarrow P$. Then $h^{\prime} \mid \partial I^{n} \times Y \approx g$ and since $\left(I^{n} \times Y, \partial I^{n} \times Y\right)$ has the homotopy extension property with respect to any space (see [W, p. 22]), $g$ extends onto $I^{n} \times Y$.

The double mapping cylinder $D M(f)$ of a map $f: X \rightarrow Y$ is the subspace $X \times I \cup M(f) \times\{0,1\}$ of $M(f) \times[0,1]$ (we consider both $X$ and $Y$ as subspaces of $M(f))$. Notice that $D M\left(\mathrm{id}_{X}\right)$ is homeomorphic to $X \times I$.

1.3. Theorem. Let $f: X \rightarrow Y$ be a map. The following conditions are equivalent:

(a) $f$ is a strong shape equivalence,

(b) given a space $Z$ containing $X$ as a closed subset, every map $g: Z \rightarrow$ $P \in \mathrm{ANR}$ extends to $Z \cup M(f)$ and every map $H: Z \times I \cup D M(f) \rightarrow P \in \mathrm{ANR}$ extends to $(Z \cup M(f)) \times I$,

(c) if $X$ is a closed subset of $Z$, then both inclusions $Z \rightarrow Z \cup M(f)$ and $Z \times I \cup D M(f) \rightarrow(Z \cup M(f)) \times I$ are shape equivalences,

(d) if $X$ is a closed subset of $Z$, then the inclusion $Z \rightarrow Z \cup M(f)$ is a strong shape equivalence,

(e) if $X$ is a closed subset of $Z$, then the inclusion $Z \rightarrow Z \cup M(f)$ is a shape equivalence,

(f) both inclusions $X \rightarrow M(f)$ and $D M(f) \rightarrow M(f) \times I$ are shape equivalences,

(g) every map $g: X \rightarrow P \in$ ANR extends to $M(f)$ and every map $H$ : $D M(f) \rightarrow P \in$ ANR extends to $M(f) \times I$.

Proof. (a) $\mapsto$ (b) If $g: Z \rightarrow P \cup$ ANR, then $g \mid X$ extends to $g^{\prime}: M(f) \rightarrow P$. By pasting $g^{\prime}$ and $g$ we get an extension $g^{\prime \prime}: Z \cup M(f) \rightarrow P$ of $g$.

Suppose $H: Z \times I \cup D M(f) \rightarrow P \in \mathrm{ANR}$ and let $q: D M\left(\mathrm{id}_{X}\right) \rightarrow D M(f)$ be the natural projection. Since $H q$ is homotopic rel. $X \times\{1\} \times\{0,1\}$ to $H^{\prime} f^{\prime}$, where $H^{\prime}: D M\left(\operatorname{id}_{Y}\right) \rightarrow P$ is a homotopy joining $H \mid Y \times\{1\} \times\{0\}$ and $H \mid Y \times\{1\} \times\{1\}$, and $f^{\prime}: D M\left(\operatorname{id}_{X}\right) \rightarrow D M\left(\operatorname{id}_{Y}\right)$ is induced by $f$, it is clear that $H$ extends onto $(Z \cup M(f)) \times I$. 
(b) $\mapsto($ c) Since $[Z \cup M(f), P] \rightarrow[Z, P]$ is onto for all $P \in$ ANR we need to show that it is injective. Suppose $g, h: Z \cup M(f) \rightarrow P$ and $g|Z \approx h| Z$. Choose a homotopy $H: Z \times I \rightarrow P$ joining $g \mid Z$ and $h \mid Z$. It induces a map $G: Z \times I \cup D M(f) \rightarrow P$ such that $G \mid M(f) \times\{0\}=g$ and $G \mid M(f) \times\{1\}=h$. Let $G^{\prime}: M(f) \times I \rightarrow P$ be an extension of $G$. Then $G^{\prime}$ is a homotopy joining $g$ and $h$.

To show that $[(Z \cup M(f)) \times I, P] \rightarrow[Z \times I \cup D M(f), P]$ is a bijection for all $P \in$ ANR it suffices to prove that for two maps $G, H:(Z \cup M(f)) \times I \rightarrow P$,

$$
G|(Z \times I \cup D M(f)) \approx H|(Z \times I \cup D M(f))
$$

implies $G \approx H$. Notice that

$$
G|(Z \times I \cup D M(f)) \approx H|(Z \times I \cup D M(f))
$$

implies

$$
G|(Z \cup M(f)) \times\{0\} \approx H|(Z \cup M(f)) \times\{0\} .
$$

However, the inclusion $(Z \cup M(f)) \times\{0\} \rightarrow(Z \cup M(f)) \times I$ is a homotopy equivalence, so $G \approx H$.

(c) $\mapsto$ (d) Suppose $H: Z \times I \rightarrow P \in$ ANR is a homotopy joining $g \mid Z$ and $h \mid Z$ for some $g, h: Z \cup M(f) \rightarrow P$. It extends to a map $G: Z \times I \cup D M(f) \rightarrow P$ such that $G \mid M(f) \times\{0\}=g$ and $G \mid M(f) \times\{1\}=h$. Let $G^{\prime}:(Z \cup M(f)) \times I \rightarrow$ $P$ be a homotopy extension of $G$ (such an extension exists by (c). Notice that the pair

$$
((Z \cup M(f)) \times I, Z \times I \cup D M(f))
$$

has the homotopy extension property with respect to any space. Indeed,

$$
(Z \times I \cup D M(f)) \times I \cup M(f) \times I \times\{0\}
$$

is a retract of

$$
(Z \times I \cup D M(f) \cup Y \times I) \times I \cup M(f) \times I \times\{0\}
$$

(because $Y \times I \times\{0\} \cup Y \times\{0,1\} \times I$ is a retract of $Y \times I \times I)$ and

$$
(Z \times I \cup D M(f) \cup Y \times I) \times I \cup M(f) \times I \times\{0\}
$$

is a retract of $(Z \cup M(f)) \times I \times I)$. Therefore we may assume that $G^{\prime}$ extends $G$. Thus the homotopy $G^{\prime}$ joins $g$ and $h$, and $G^{\prime} \mid Z \times I=G$.

$(\mathrm{d}) \rightarrow($ e) Obvious.

(e) $\rightarrow$ (f) Using $Z=X \times I \cup M(f) \times\{0\}$ we infer that the inclusion $X \times$ $I \cup M(f) \times\{0\} \rightarrow D M(f)$ is a shape equivalence. Therefore the inclusion $D M(f) \rightarrow M(f) \times I$ is a shape equivalence. Using $Z=X$ we get that $X \rightarrow$ $M(f)$ is a shape equivalence.

$(\mathrm{f}) \mapsto(\mathrm{g})$ Obvious in view of the fact that both $(M(f), X)$ and $(M(f) \times$ $I, D M(f))$ have the homotopy extension property with respect to any space.

(g) $\mapsto\left(\right.$ a) Suppose $H: D M\left(\mathrm{id}_{X}\right) \rightarrow P \in$ ANR is a homotopy joining $g f$ and $h f$, where $g, h: Y \rightarrow P$. It induces a map $G: D M(f) \rightarrow P$ such that $G|Y \times\{0\}=g, G| Y \times\{1\}=h$. Let $G^{\prime}: M(f) \times I \rightarrow P$ be an extension of 
$G$. Using the projection $p: X \times I \times I \rightarrow M(f) \times I$ and a strong deformation retraction of $X \times I \times I$ onto $X \times\{1\} \times I$ one gets that $H$ is homotopic rel. $X \times\{1\} \times\{0,1\}$ to $H^{\prime} \cdot\left(f \times \mathrm{id}_{I}\right)$ for some homotopy $H^{\prime}: D M\left(\operatorname{id}_{Y}\right) \rightarrow P$ joining $g$ and $h$. Thus $f$ is a strong shape equivalence.

In case where $f=i$ is the inclusion $i: A \rightarrow X$ Theorem 1.3(f) has the following simple interpretation:

1.4. Corollary. Suppose $A$ is a subspace of $X$. The inclusion $i: A \rightarrow X$ is a strong shape equivalence iff both $i$ and the inclusion

$$
j: X \times\{0,1\} \cup A \times I \rightarrow X \times I
$$

are shape equivalences.

1.5. Corollary. If $f: X \rightarrow Y$ is a homotopy equivalence, then it is a strong shape equivalence.

Proof. $X$ is a strong deformation retract of $M(f)$ (see [W, Corollary 5.10, p. 25]). Therefore $Y \times\{0\}$ is a strong deformation retract of $D M(f)$ and both $X \rightarrow M(f)$ and $D M(f) \rightarrow M(f) \times I$ are homotopy equivalences.

1.6. Corollary. If $f^{\prime}: X \rightarrow Y$ is homotopic to a strong shape equivalence $f$, then $f^{\prime}$ is a strong shape equivalence.

Proof. $M\left(f^{\prime}\right)$ is homotopy equivalent to $M(f)$ rel. $X$ (see $\left[\mathrm{F}_{2}\right]$ ). Therefore for every space $Z$ containing $X$ as a closed set $Z \cup M(f)$ and $Z \cup M\left(f^{\prime}\right)$ are homotopy equivalent rel. $Z$. By Theorem 1.3(e), $f^{\prime}$ is a strong shape equivalence.

1.7. Proposition. If $f: X \rightarrow Y$ and $g: Y \rightarrow Z$ are strong shape equivalences, then $g \cdot f$ is also a strong shape equivalence.

Proof. Obviously $g f$ is a shape equivalence. Suppose $a, b: Z \rightarrow P \in$ ANR are two maps and $H: X \times I \rightarrow P$ is a homotopy joining agf and $b g f$. Then there is a homotopy $H^{\prime}: Y \times I \rightarrow P$ joining $a g$ and $b g$ such that $H \approx H^{\prime}(f \times$ id $)$ rel. $X \times\{0,1\}$. Again, there is a homotopy $H^{\prime \prime}: Z \times I \rightarrow P$ joining $a$ and $b$ such that

$$
H^{\prime} \approx H^{\prime \prime}(g \times \text { id }) \text { rel. } Y \times\{0,1\} .
$$

Then $H^{\prime \prime}(g f \times$ id $) \approx H$ rel. $X \times\{0,1\}$.

1.8. Theorem. Suppose $f: X \rightarrow Y$ and $g: Y \rightarrow Z$ are maps such that $g \cdot f$ is a strong shape equivalence. If one of $f$ and $g$ is a shape equivalence, then both $f$ and $g$ are strong shape equivalences.

Proof. Observe that both $f$ and $g$ are shape equivalences. First let us show that $f$ is a strong shape equivalence. Suppose $H: D M(f) \rightarrow P \in$ ANR. Since $Y \rightarrow M(g)$ is a shape equivalence, there is an extension

$$
H^{\prime}: X \times I \cup M(f) \times\{0,1\} \cup M(g) \times\{0,1\} \rightarrow P
$$


of $H$. Since $M(f) \cup M(g)$ is homotopy equivalent of $M(g f)$ rel. $X$ (see $\left[\mathrm{F}_{2}\right]$ ) and $g f$ is a strong shape equivalence, $H^{\prime}$ extends onto $(M(f) \cup M(g)) \times I$. In particular, $H$ extends onto $M(f) \times I$. By Theorem 1.3(g) $f$ is a strong shape equivalence.

Suppose $H: Y \times I \rightarrow P \in$ ANR is a homotopy joining $g a$ and $g b$, where $a, b: Z \rightarrow P$. Since $g f$ is a strong shape equivalence, there is a homotopy $H^{\prime \prime}: Z \times I \rightarrow P$ joining $a$ and $b$ such that

$$
H^{\prime \prime}(g f \times \text { id }) \approx H(f \times \text { id }) \text { rel. } X \times\{0,1\} .
$$

Construct

$$
G: Y \times\left(\partial I^{2}\right) \rightarrow P
$$

by $G(y, 0, t)=H(y, t), G(y, 1, t)=H^{\prime \prime}(g(y), t), G(y, t, 0)=a g(y)$ and $G(y, t, 1)=b g(y)$ for all $y \in Y$ and $t \in I$. Then $G(f \times$ id $): X \times\left(\partial I^{2}\right) \rightarrow P$ extends onto $X \times I^{2}$, so by Proposition 1.2, $G$ extends onto $Y \times I^{2}$. This means that

$$
H \approx H^{\prime \prime}(g \times \text { id }) \text { rel } . Y \times\{0,1\} .
$$

1.9. Corollary. Suppose the inclusion $f: X \rightarrow Y$ is a shape equivalence. If $f$ has either left or right homotopy inverse, then it is a strong shape equivalence.

Proof. Suppose $g f \approx \mathrm{id}_{X}$ (resp. $f g \approx \mathrm{id}_{Y}$ ). By Corollary 1.6, $g f$ (resp. $f g$ ) is a strong shape equivalence and Theorem 1.8 implies that $f$ is a strong shape equivalence.

1.10. Corollary. Suppose $f: X \rightarrow Y$ is a shape equivalence. If $X$ has the homotopy type of an ANR, then $f$ is a strong shape equivalence.

Proof. There is a map $g: Y \rightarrow X$ such that $g f$ is homotopic to $\operatorname{id}_{X}$.

1.11. Theorem. Let $f: X \rightarrow Y$ be a map of topological spaces. The following conditions are equivalent:

(a) $f$ is a strong shape equivalence,

(b) for all $Z$ of the homotopy type of an ANR and containing $X$ as a closed subset the inclusion $Z \rightarrow Z \cup M(f)$ is a shape equivalence.

Proof. It suffices to show (b) $\rightarrow($ a) . Suppose $g: X \rightarrow P \in$ ANR. Put $Z=$ $M(g)$. Since $Z \rightarrow Z \cup M(f)$ is a shape equivalence and $(Z \cup M(f), Z)$ satisfies the homotopy extension property with respect to all spaces (see [W, p. 23]), the natural map $Z \rightarrow P$ extends over $Z \cup M(f)$. Then the restriction $g^{\prime}$ of the map $Z \cup M(f) \rightarrow P$ to $M(f)$ is an extension of $g$. Now suppose $g$, $h: Y \rightarrow P \in$ ANR and $g f$ is homotopic to $h f$. Put $Z=M(g f)$. Then on $Z \cup M(f)$ we can define two maps: $g^{\prime}: Z \cup M(f) \rightarrow P$ and $h^{\prime}: Z \cup M(f) \rightarrow P$ such that both $g^{\prime} \mid Z$ and $h^{\prime} \mid Z$ are natural projections and $g^{\prime}\left|Y=g, h^{\prime}\right| Y=h$. Since the inclusions $Z \rightarrow Z \cup M(f)$ is a shape equivalence and $g^{\prime}\left|Z=h^{\prime}\right| Z$, we conclude that $g^{\prime}$ is homotopic to $h^{\prime}$. Restricting the homotopy to $Y$ one gets $g$ is homotopic to $h$. 
Thus $f: X \rightarrow Y$ is a shape equivalence. It remains to show that the inclusion $X \rightarrow D M(f)$ is a shape equivalence (in such a case $D M(f) \rightarrow M(f) \times I$ must be a shape equivalence). Suppose $Z$ contains $X$ as a closed subset and has the homotopy type of an ANR. Since $Z \rightarrow Z \cup M(f)$ is a shape equivalence, by Corollary 1.10 it is a strong shape equivalence. Applying Theorem 1.3(d), one gets that the inclusion $Z \cup M(f) \rightarrow Z \cup D M(f)$ is a strong shape equivalence. Thus the inclusion $Z \rightarrow Z \cup D M(f)$ is a shape equivalence. By putting $X^{\prime}=X$ and $Y^{\prime}=D M(f)$ in the first part of the proof we get that $X \rightarrow D M(f)$ is a shape equivalence.

1.12. Proposition. If $f: X \rightarrow Y$ is a strong shape equivalence and $Z$ is a compactum, then $f \times \mathrm{id}: X \times Z \rightarrow Y \times Z$ is a strong shape equivalence.

Proof. For any $P \in$ ANR the space $P^{Z}$ is also an ANR if $Z$ is compact metrizable (see [M-S, p. 38]). Therefore $f_{*}:\left[Y, P^{Z}\right] \rightarrow\left[X, P^{Z}\right]$ is a bijection which is equivalent to saying that

$$
(f \times \mathrm{id})_{*}:[Y \times Z, P] \rightarrow[X \times Z, P]
$$

is a bijection. Thus $f \times$ id is a shape equivalence. Similarly one shows that $D M(f \times$ id $) \rightarrow M(f \times$ id $) \times I$ is a shape equivalence.

1.13. Theorem. Suppose a map $f:(X, x) \rightarrow(Y, y)$ is a pointed shape equivalence. If $X, Y$ are connected and $\operatorname{def}-\operatorname{dim} X$, def-dim $Y$ are finite, then $f$ is a strong shape equivalence.

Proof. As in the proof of Theorem 7.1 in [D-S $]$ one shows that for every space $Z$ containing $X$ as a closed subset, the inclusion $i:(Z, x) \rightarrow(Z \cup M(f), x)$ induces isomorphisms of all pro-homotopy groups. Applying that to the case $Z=X \times I \cup M(f) \times\{0\}$ one gets that the inclusion $M(f) \times\{0\} \rightarrow D M(f)$ is a shape equivalence (this is so in view of def-dim $(D M(f)) \leq \operatorname{def}-\operatorname{dim} X+1$, see the proof of Theorem 5.3.8 in [D-S ${ }_{2}$, p. 73]). Therefore the inclusion $D M(f) \rightarrow$ $M(f) \times I$ is a shape equivalence.

1.14. Theorem. The family SSE of strong shape equivalences admits a calculus of left fractions. That is, the following conditions hold:

(a) SSE is closed under composition and contains all identity morphisms,

(b) given $u: A \rightarrow Y$ and $s: A \rightarrow X$ with $s \in \mathrm{SSE}$ there exist $u^{\prime}: X \rightarrow Z$ and $s^{\prime}: Y \rightarrow Z$ with $s^{\prime} u \approx u^{\prime} s$ and $s^{\prime} \in \mathrm{SSE}$,

(c) given $u, v: X \rightarrow Y$ and $s \in \mathrm{SSE}$ such that $u s \approx v s$, there exists $t \in \mathrm{SSE}$ such that $t u \approx t v$.

Proof. (a) follows from Proposition 1.7.

(b) Put $Z=M(s) \cup M(u)$ and $u^{\prime}, s^{\prime}$ are natural maps. Since $M(u) \rightarrow Z$ is a strong shape equivalence (Theorem 1.3(d)), $s^{\prime}: Y \rightarrow Z$ is a strong shape equivalence.

(c) Any homotopy joining $u s$ and $v s$ induces a map $H: D M(s) \rightarrow Y$ such that $H \mid X \times\{0\}=u$ and $H \mid X \times\{1\}=v$ Let $p: D M(s) \rightarrow X$ be the 
natural projection. By Theorem 1.3 and Theorem $1.8, p$ is a strong shape equivalence. Consider the natural inclusion $t: Y \rightarrow Z=M(H) \cup M(p)$. It is a strong shape equivalence by Theorem 1.3(d) and Proposition 1.7 (applied to $Y \rightarrow M(H) \rightarrow M(H) \cup M(p))$. It is clear that $t u \approx t v$.

Theorem 1.14 allows to define a category of left fractions (SSE) ${ }^{-1}$ HTOP (see [G-Z]) by formally inverting all homotopy classes of strong shape equivalences. In the compact metrizable case it was described by Calder-Hastings [C-H] and Cathey $[\mathrm{C}]$.

\section{STRONG SHAPE EQUiVALENCES BETWEEN PARACOMPACT SPACES}

In case of paracompact spaces certain of the above results have a simpler form. This is because every $P \in$ ANR has the homotopy type of a topologically complete metrizable space $P^{\prime} \in$ ANR which is an ANE for paracompact spaces (see [D-K, p. 211]). Therefore in all questions regarding shape and strong shape equivalences we may consider only metrizable $P \in \mathrm{ANE}$ (paracompact). In particular every pair $(X, A), X$ paracompact and $A$ closed in $X$, has the homotopy extension property with respect to $P$.

2.0. Proposition. $M(f)$ is paracompact for any map $f: X \rightarrow Y$ of paracompact spaces.

Proof. Notice that one-point subsets in $M(f)$ are closed. Suppose $A$ is closed in $M(f)$ and $g: A \rightarrow I$ is continuous. First extend it to $g^{\prime}: A \cup Y \rightarrow I$. Let $p: X \times I \oplus Y \rightarrow M(f)$ be the projection. Since $X \times I \oplus Y$ is normal, $g p$ : $p^{-1}(A \cup Y) \rightarrow I$ extends onto $X \times I \oplus Y$. That extension induces an extension $g^{\prime \prime}: M(f) \rightarrow I$ of $g$. Thus $M(f)$ is normal. To show it is paracompact it suffices to prove that every open cover $U$ of $M(f)$ has an open $\sigma$-locally finite refinement [E, Theorem 4, p. 210]. Since $M(f)-Y$ is paracompact, we need to find a locally finite refinement $U^{\prime}$ of $U$ covering $Y$. First find a locally finite refinement $V$ of $U \cap Y$. $U^{\prime}$ is constructed as follows: for $A$ in $V$ choose $A^{\prime}$ in $U$ containing $A$, then take $p^{-1}(A) \cap A^{\prime}$. Since $p^{-1}(V)$ is locally finite, so is $U^{\prime}$.

2.1. Theorem. Suppose $f: X \rightarrow Y$ is a map of paracompact spaces and $X$ is a closed subset of a paracompact space $Z$. Then the projection $p: Z \cup M(f) \rightarrow$ $Y \cup_{f} Z$ is a strong shape equivalence if $Y \cup_{f} Z$ is paracompact.

Proof. Let $q: Z \rightarrow Y \cup_{f} Z$ be the natural projection. Then $Z \cup M(f)$ is contained in $M(q)$. It suffices to show that the inclusion $i: Z \cup M(f) \rightarrow M(q)$ is a strong shape equivalence. Suppose $g: Z \cup M(f) \rightarrow P \in \mathrm{ANE}$ (paracompact) is a map. There is a closed neighborhood $U$ of $X$ in $Z$ and an extension $g^{\prime}: Z \cup M(q \mid U) \rightarrow P$ of $g$. Since $M(q)-Z \cup M(q \mid$ int $U)$ is homeomorphic to $(Z-$ int $U) \times I$, the homotopy extension property for $(Z-\operatorname{int} U, U-\operatorname{int} U)$ implies the existence of an extension $g^{\prime \prime}: M(q) \rightarrow P$ of $g$. Similarly one can show that every map $D M(i) \rightarrow P$ extends onto $M(i) \times I$. 
2.2. Corollary. Suppose $f: X \rightarrow Y$ is a map of paracompact spaces and $X$ is a closed subset of a paracompact space $Z$. If $f$ is a strong shape equivalence, then the projection $Z \rightarrow Y \cup_{f} Z$ is a strong shape equivalence if $Y \cup_{f} Z$ is paracompact.

Proof. Apply Theorem 1.3(d) and Proposition 1.7.

2.3. Lemma. Suppose $A$ is a closed subset of a paracompact space $X$ and

$$
g: A \times I \cup X \times\{0,1\} \rightarrow P \in \mathrm{ANE} \text { (paracompact) }
$$

is a $r$ such that $g \mid A \times\{0\} \approx$ const. Then $g$ extends onto $X \times I$ if one of the following conditions holds:

(a) $i: A \rightarrow X$ is a shape equivalence,

(b) $X / A$ is of trivial shape.

Proof. Suppose $i: A \rightarrow X$ is a shape equivalence. Since $g \mid X \times\{0\} \approx$ const, then $g \mid X \times\{1\} \approx$ const. We may simply assume that $g \mid X \times\{0\}=$ const and $g \mid X \times\{1\}=$ const. Then $g$ factors as $g=g^{\prime} p$, where $p: A \times I \cup X \times$ $\{0,1\} \rightarrow \Sigma A$ is the natural projection (notice that the suspension $\Sigma A$ of $A$ is $A \times I \cup X \times\{0,1\}$ with $X \times\{0\}$ and $X \times\{1\}$ contracted to points). Since $\Sigma A \rightarrow \Sigma X$ is a shape equivalence $([\Sigma A, R]=[A, \Omega R]=[X, \Omega R]=[\Sigma X, R]$ for any $R \in$ ANR), $g^{\prime}$ extends to $g^{\prime \prime}: \Sigma X \rightarrow P$. Then $g^{\prime \prime} p^{\prime}$ extends $g$, where $p^{\prime}: X \times I \rightarrow \Sigma X$ is the natural extension of $p$.

If $X / A$ is of trivial shape, we homotop $g$ to $g^{\prime}$ with $g^{\prime} \mid A \times I=$ const (use that $(X / A, *)$ is of trivial shape; see [M-S, Lemma 4, p. 104]). Then $g^{\prime}$ factors as $g^{\prime}=g^{\prime \prime}(p \times$ id $)$, where $p: X \rightarrow X / A$ is the projection and

$$
g^{\prime \prime}:(X / A) \times\{0,1\} \cup(A / A) \times I \rightarrow P .
$$

Since the inclusion $A / A \rightarrow X / A$ is a strong shape equivalence (see Corollary 1.10), $g^{\prime \prime}$ extends onto $(X / A) \times I$. Therefore $g$ extends onto $X \times I$.

2.4. Corollary. If $A$ is a closed subset of a paracompact space $X$ and the inclusion $i: A \rightarrow X$ is a shape equivalence, then $X / A$ is of trivial shape.

Proof. Suppose $g: X / A \rightarrow P \in$ ANE (paracompact) is a map. It induces a map $G: A \times I \cup X \times\{0,1\} \rightarrow P$ such that

$$
G(A \times I \cup X \times\{1\})=g(A / A)
$$

and $G \mid X \times\{0\}=g p$, where $p: X \rightarrow X / A$ is the projection. Then any extension $G^{\prime}: X \times I \rightarrow P$ of $G$ induces a homotopy between $g$ and a constant map.

2.5. Corollary. If $f: X \rightarrow Y$ is a shape equivalence of paracompact spaces, then $(M(f) \times I) / D M(f)$ is of trivial shape.

Proof. First $(M(f) \times I) / Y \times\{0\}$ is of trivial shape. Furthermore, $D M(f) / Y \times$ $\{0\}$ is of trivial shape since it is homeomorphic to $M(f) / X$. Thus

$$
(M(f) \times I) / D M(f)=((M(f) \times I) / Y \times\{0\}) /(D M(f) / Y \times\{0\})
$$

is of trivial shape. 
2.6. Corollary. If $f: X \rightarrow Y$ is a shape equivalence of paracompact spaces, then every map $g: D M(f) \rightarrow P \in \operatorname{ANE}$ (paracompact) such that $g \mid Y \times\{0\} \approx$ const extends onto $M(f) \times I$ uniquely up to homotopy rel. $D M(f)$.

Proof. Notice that $g \approx$ const. Indeed, we may assume $g \mid Y \times\{0\}=$ const and since $g$ factors through $D M(f) / Y \times\{0\}$ which is of trivial shape since it is homeomorphic to $M(f) / X, g \approx$ const. Therefore $g$ extends onto $M(f) \times I$. Lemma 2.3 implies that every two such extensions are homotopic rel. $D M(f)$.

2.7. Theorem. If $f: X \rightarrow Y$ is a closed surjective map of paracompact spaces such that for each closed set $B$ of $Y$ the map $f \mid f^{-1}(B): f^{-1}(B) \rightarrow B$ is a shape equivalence, then $f$ is a strong shape equivalence.

Proof. We need to show that every map $g: D M(f) \rightarrow P \in$ ANE (paracompact) extends onto $M(f) \times I$ (Theorem 1.3(g)). Let $p: M(f) \times I \rightarrow Y$ be the natural projection. Notice that $p^{-1}(y) \cap D M(f)=\Sigma f^{-1}(y)$ for each $y$ in $Y$. Therefore $g \mid p^{-1}(y) \cap D M(f) \approx$ const for each $y$ in $Y$, and $g$ extends onto some neighborhood of $p^{-1}(y) \cap D M(f)$ in $M(f) \times I$. Choose a neighborhood $V_{y}$ of $y$ in $Y$ such that $g$ extends onto $D M(f) \cup p^{-1}\left(V_{y}\right)$. Then choose a locally finite cover $\left\{A_{s}\right\}_{s \in S}$ consisting of closed sets which is a refinement of $\left\{V_{y}\right\}_{y \in Y}$. For each $s$ in $S$ choose a map

$$
g_{s}: D M\left((f) \cup p^{-1}\left(V_{y}\right) \rightarrow P\right.
$$

extending $g$ such that $g_{s}\left(A_{s} \times\{0\}\right) \approx$ const. If $g^{\prime}: D M(f) \cup p^{-1}(A) \rightarrow P$ is an extension of $g$ ( $A$ closed in $Y$ ) and $s \in S$, then

$$
g^{\prime}\left|p^{-1}\left(A \cap A_{s}\right) \approx g_{s}\right| p^{-1}\left(A \cap A_{s}\right) \text { rel. } D M(f) \cap p^{-1}\left(A \cap A_{s}\right)
$$

(see Corollary 2.6). Since $g_{s} \mid p^{-1}\left(A \cap A_{s}\right)$ extends onto $p^{-1}\left(A_{s}\right), g^{\prime}$ extends onto $p^{-1}\left(A_{s}\right)$. Therefore we have an extension

$$
g^{\prime \prime}: D M(f) \cup p^{-1}\left(A \cup A_{s}\right) \rightarrow P
$$

of $g$. By well-ordering $S$ and by the transfinite induction we can construct an extension $G: M(f) \times I \rightarrow P$ of $g$.

2.8. Corollary. Suppose $f: X \rightarrow Y$ is a map of paracompact spaces, $A$ and $B$ are closed subsets of $X=A \cup B, A^{\prime}$ and $B^{\prime}$ are closed subsets of $Y=A^{\prime} \cup B^{\prime}$. If $f\left|A: A \rightarrow A^{\prime}, f\right| B: B \rightarrow B^{\prime}$ and $f \mid A \cap B: A \cap B \rightarrow A^{\prime} \cap B^{\prime}$ are strong shape equivalences, then $f$ is a strong shape equivalence.

Proof. Replacing $Y$ by $M(f)$ we reduce Corollary 2.8 to the case where $f$ is an inclusion, $A=X \cap A^{\prime}$ and $B=X \cap B^{\prime}$. Then $X \rightarrow X \cup B^{\prime}$ and $A \rightarrow A \cup\left(A^{\prime} \cap B^{\prime}\right) \rightarrow A^{\prime}$ are strong shape equivalences (use Propositions 1.71.8). Consequently $A \cup B^{\prime}=X \cup B^{\prime} \rightarrow A^{\prime} \cup B^{\prime}=Y$ is a strong shape equivalence which completes the proof.

2.9. Theorem. Suppose $f: X \rightarrow Y$ is a map of paracompact spaces and $\mathbf{S}$ is a locally finite cover of $Y$ consisting of closed sets. If for arbitrary elements 
$A_{1}, \ldots, A_{k}$ of $\mathbf{S}$ the map

$$
f \mid f^{-1}\left(\cap A_{i}\right): f^{-1}\left(\cap A_{i}\right) \rightarrow \cap A_{i}
$$

is a strong shape equivalence, then $f$ is a strong shape equivalence.

Proof. Corollary 2.8 implies Theorem in case $\mathbf{S}$ is finite. First we are going to show that every map $g: X \rightarrow P \in$ ANE (paracompact) extends onto $M(f)$. For each $A$ in $\mathbf{S}$ choose a map

$$
g_{A}: X \cup M\left(f \mid f^{-1}(A)\right) \rightarrow P
$$

extending $g$. If

$$
g^{\prime}: X \cup M\left(f \mid f^{-1}\left(\bigcup \mathbf{S}^{\prime}\right)\right) \rightarrow P
$$

is an extension of $g\left(\mathbf{S}^{\prime}\right.$ a subset of $\left.\mathbf{S}\right)$ and $B \in \mathbf{S}$, then

$$
g^{\prime}\left|M\left(f^{-1}\left(B \cap\left(\bigcup \mathbf{S}^{\prime}\right)\right)\right) \approx g_{\beta}\right| M\left(f^{-1}\left(B \cap\left(\bigcup \mathbf{S}^{\prime}\right)\right)\right) \text { rel. } f^{-1}\left(B \cap\left(\bigcup \mathbf{S}^{\prime}\right)\right)
$$

(this is because the inclusion

$$
f^{-1}\left(B \cap\left(\bigcup \mathbf{S}^{\prime}\right)\right) \rightarrow M\left(f^{-1}\left(B \cap\left(\bigcup \mathbf{S}^{\prime}\right)\right)\right)
$$

is a strong shape equivalence). Since $g_{B} \mid M\left(f^{-1}\left(B \cap\left(\bigcup \mathbf{S}^{\prime}\right)\right)\right)$ extends onto $M\left(f^{-1}(B)\right), g^{\prime}$ extends onto $X \cup M\left(f^{-1}(B)\right)$. Therefore we have an extension

$$
g^{\prime \prime}: X \cup M\left(f^{-1}\left(\left(\bigcup \mathbf{S}^{\prime}\right) \cup B\right)\right) \rightarrow P
$$

of $g$. By well-ordering $S$ and by the transfinite induction we can construct an extension $G: M(f) \rightarrow P$ of $g$.

Let $p: M(f) \times I \rightarrow Y$ be the natural projection. Notice that

$$
p^{-1}(A) \cap D M(f)=D M\left(f \mid f^{-1}(A)\right)
$$

for each closed set $A$ of $Y$. Since the inclusion

$$
D M\left(f \mid f^{-1}(A)\right) \rightarrow M\left(f \mid f^{-1}(A)\right) \times I
$$

is a strong shape equivalence for each $A \in \mathbf{S}$, we infer that

$$
p \mid D M\left(f \mid f^{-1}(A)\right): D M\left(f \mid f^{-1}(A)\right) \rightarrow A
$$

is a strong shape equivalence. By the first part of the proof every map

$$
g: D M(f) \rightarrow P \in \mathrm{ANE} \text { (paracompact) }
$$

factors up to homotopy through $Y$. Therefore it extends onto $M(f) \times I$.

2.10. Theorem. Suppose $X, Y$ and $B$ are paracompact spaces and $p_{1}: X \rightarrow$ $B, p_{2}: Y \rightarrow B$ are closed surjective maps. Suppose $f: X \rightarrow Y$ is a closed map such that for each $b$ in $B$,

$$
f \mid p_{1}^{-1}(b): p_{1}^{-1}(b) \rightarrow p_{2}^{-1}(b)
$$


is a strong shape equivalence. If Ind $B$ (large inductive dimension of $B$ ) is finite (or locally finite), then $f$ is a strong shape equivalence.

Proof. By induction on $n=\operatorname{Ind} B$. If $n=-1$ it is obvious. Suppose Theorem 2.10 holds for all cases where Ind $B<n, n \geq 0$. Consider the case with Ind $B=n$. Suppose

$$
g: X \rightarrow P \in \mathrm{ANE} \text { (paracompact) }
$$

is a map. Our goal is to extend $g$ onto $M(f)$. Given $b \in B$ one can extend $g$ onto $X \cup M\left(f \mid p_{1}^{-1}(b)\right)$ and then onto some neighborhood $U_{b}$ of $X \cup M\left(f \mid p_{1}^{-1}(b)\right)$ in $M(f)$. Since each $U_{b}$ contains $M\left(f \mid p_{1}^{-1}\left(V_{b}\right)\right)$ for some neighborhood $V_{b}$ of $b$ in $B$, we may assume that $B=\bigcup \mathbf{S}$, where $\mathbf{S}$ is a locally finite cover consisting of closed sets and for each $A$ in $\mathbf{S}$ there is an extension $g_{A}: X \cup M\left(f \mid p_{1}^{-1}(A)\right) \rightarrow P$ of $g$. If

$$
g^{\prime}: X \cup M\left(f \mid p_{1}^{-1}\left(\bigcup \mathbf{s}^{\prime}\right)\right) \rightarrow P
$$

is an extension of $g\left(\mathbf{S}^{\prime}\right.$ a subset of $\left.\mathbf{S}\right)$ and $A \in \mathbf{S}$, then first extend $g^{\prime}$ to

$$
g^{\prime \prime}: X \cup M\left(f \mid p_{1}^{-1}(U)\right) \rightarrow P,
$$

where $U$ is a neighborhood of $U \mathbf{S}^{\prime}$ in $B$ and then choose a neighborhood $V$ of $U \mathbf{S}^{\prime}$ in $U$ with $\operatorname{Ind} \operatorname{Fr} V \leq n-1$ and $\operatorname{cl}(V)$ contained in $U$. Let $C=(\operatorname{Fr} V) \cap A$. Now we have two extensions

$$
g_{A} \mid M\left(f \mid p_{1}^{-1}(C)\right) \text { and } g^{\prime \prime} \mid M\left(f \mid p_{1}^{-1}(C)\right)
$$

of $g$. By the inductive assumption,

$$
f \mid p_{1}^{-1}(C): p_{1}^{-1}(C) \rightarrow p_{2}^{-1}(C)
$$

is a strong shape equivalence, so

$$
g_{A}\left|M\left(f \mid p_{1}^{-1}(C)\right) \approx g^{\prime \prime}\right| M\left(f \mid p_{1}^{-1}(C)\right) \text { rel. } p_{1}^{-1}(C) .
$$

Since $g_{A} \mid M\left(f \mid p_{1}^{-1}(C)\right)$ extends onto $M\left(f \mid p_{1}^{-1}(A)\right), g^{\prime \prime}$ extends onto $X \cup$ $M\left(f \mid p_{1}^{-1}(A)\right)$. Therefore we have an extension

$$
G: X \cup M\left(f \mid p_{1}^{-1}\left(A \cup\left(\bigcup \mathbf{S}^{\prime}\right)\right)\right) \rightarrow P
$$

of $g$. By well-ordering $S$ and by the transfinite induction we can construct an extension $G: M(f) \rightarrow P$ of $g$.

Let $i: D M(f) \rightarrow M(f) \times I$ be the inclusion and $p: M(f) \times I \rightarrow Y$ the natural projection. Notice that

$$
p^{-1}\left(p_{2}^{-1}(b)\right) \cap D M(f)=D M\left(f \mid p_{1}^{-1}(b)\right)
$$

for each point $b$ of $B$. Since the inclusion

$$
D M\left(f \mid p_{1}^{-1}(b)\right) \rightarrow M\left(f \mid p_{1}^{-1}(b)\right) \times I=p^{-1}\left(p_{2}^{-1}(b)\right)
$$


is a strong shape equivalence for each $b \in B$, we infer from the first part of the proof that every map

$$
g: D M(f) \rightarrow P \in \operatorname{ANE}(\text { paracompact })
$$

extends onto $M(f) \times I$.

2.11. Theorem. Suppose $X$ and $Y$ are paracompact spaces, $X=\bigcup_{n=1}^{\infty} X_{n}$, $X_{n}$ closed in $X$ and $X_{n} \subset \operatorname{int} X_{n+1}, Y=\bigcup_{n=1}^{\infty} Y_{n}, Y_{n}$ closed in $Y$ and $Y_{n} \subset$ int $Y_{n+1}$. If $f: X \rightarrow Y$ is a map such that for each $n, f_{n}=f \mid X_{n}: X_{n} \rightarrow Y_{n}$ is a strong shape equivalence, then $f$ is a strong shape equivalence.

Proof. Since $X \rightarrow X \cup M\left(f_{n}\right)$ is a shape equivalence for each $n$, the inclusion $X \cup M\left(f_{n}\right) \rightarrow X \cup M\left(f_{n+1}\right)$ is a shape equivalence for each $n$. Given

$$
g: X \rightarrow P \in \mathrm{ANE} \text { (paracompact) }
$$

we can keep extending $g$ to $X \cup M\left(f_{1}\right)$ and then from $X \cup M\left(f_{n}\right)$ to $X \cup$ $M\left(f_{n+1}\right)$. Therefore $g$ extends onto $M(f)$. Applying the same step to the inclusion $D M(f) \rightarrow M(f) \times I$ one gets that any

$$
g: D M(f) \rightarrow P \in \operatorname{ANE}(\text { paracompact })
$$

extends onto $M(f) \times I$.

\section{Fibrant Diagrams}

Given a finite diagram $D$ in TOP with commutation relations and no loops (i.e., the beginning and end of a chain of arrows are always distinct) one can consider for each vertex $a$ of $D$ the subdiagram $D(a)$ consisting of vertices reachable from $a$ (but not $a$ ) and arrows between them. The space at the vertex $a$ is denoted by $X(a)$ and the map (if it exists) from $X(b)$ to $X(a)$ is denoted by $p_{a, b}$.

3.1. Definition. Given a diagram $D, \lim D$ is the subspace $\left\{\left\{x_{a}\right\} \mid p_{a, b}\left(x_{b}\right)=\right.$ $\left.x_{a}\right\}$ of the cartesian product $\Pi X(a)$.

3.2. Definition. $D^{\prime}$ is a full subdiagram of $D$ if for every vertex $a$ of $D^{\prime}, D^{\prime}$ contains $D(a)$ and all the arrows beginning at $a$.

3.3. Definition. $D$ is called a fibrant diagram if the projection $\lim D^{\prime} \rightarrow \lim D^{\prime \prime}$ is a fibration for each pair of full subdiagrams $D^{\prime \prime} \subset D^{\prime}$ of $D$.

3.4. Proposition. If $D$ is a fibrant diagram, then for each vertex $a$ of $D$ the natural projections $\lim D \rightarrow X(a)$ and $X(a) \rightarrow \lim D(a)$ are fibrations. In particular, all the arrows in $D$ represent fibrations.

Proof. Let $D^{\prime}(a)$ be the union of $D(a)$ and all the arrows beginning at $a$. Then $\lim D^{\prime}(a)$ is simply $X(a)$ and $p_{b, a}: X(a) \rightarrow X(b)$ corresponds to $\lim D^{\prime}(a) \rightarrow$ $\lim D^{\prime}(b)$.

3.5. Theorem. Suppose $D$ is a diagram in TOP such that for each vertex a of $D$ the projection $X(a) \rightarrow \lim D(a)$ is a fibration. If each proper full subdiagram 
$D^{\prime}$ of $D$ is fibrant, then $D$ is fibrant. If $D$ is a fibrant diagram in ANRs, then $\lim D$ is an $A N R$.

Proof. The proof is by induction on the number $k$ of vertices in $D$. The subsequent lemma takes care of cases with $k \leq 3$.

3.6. Lemma. If $D$ is the diagram in TOP

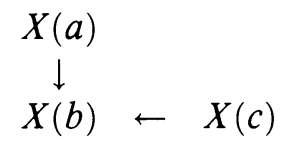

and $p_{b, c}: X(c) \rightarrow X(b)$ is a fibration, then $\lim D \rightarrow X(a)$ is a fibration. If, in addition, all three spaces $X(a), X(b)$ and $X(c)$ are $A N R s$, then $\lim D$ is an ANR.

Proof of Lemma 3.6. The first part of Lemma 3.6 follows from the fact that the pullback of a fibration is a fibration (see [W, Theorem 7.21, p. 37]).

It remains to show that $\lim D$ is an ANR, if all three spaces $X(a), X(b)$ and $X(c)$ are ANRs. We are going to produce an open neighborhood $U$ of $\lim D$ in $X(a) \times X(b) \times X(c)$ and a retraction $r: U \rightarrow \lim D$.

Let $p: X(a) \times X(b) \times X(c) \rightarrow X(a)$ and $q: X(a) \times X(b) \times X(c) \rightarrow X(c)$ be projections. Since $p_{b, a} p\left|\lim D=p_{b, c} q\right| \lim D$, there is an open neighborhood $U$ of $\lim D$ in $X(a) \times X(b) \times X(c)$ such that $p_{b, a} p \mid U$ is homotopic to $p_{b, c} q \mid U$ rel. $\lim D$. Let $H: U \times I \rightarrow X(b)$ be a homotopy joining $p_{b, c} q$ and $p_{b, a} p$ with $H \mid\{x\} \times I$ being constant for $x$ in $\lim D$. Using the fact that $p_{b, c}$ is a fibration and Corollary 2.4 in [D, p. 397] one can find $H^{\prime}: U \times I \rightarrow X(c)$ with $H=p_{b, c} H^{\prime}, H^{\prime}$ starts at $q \mid U$ and stays fixed on $\lim D$. Consider $h: U \rightarrow X(c)$ defined by $h(x)=H^{\prime}(x, 1)$. Then $p_{b, c} h=p_{b, a} p \mid U$ and therefore $r: U \rightarrow \lim D$ defined by $r(x)=\left(p(x), p_{b, a} p(x), h(x)\right)$ is a retraction.

Obviously, Theorem 3.5 is valid for all diagrams with the number of vertices at most 2. Suppose $n \geq 3$ is an integer such that Theorem 3.5 holds for all diagrams with the number of vertices less than $n$. Consider a diagram $D$ with $n$ vertices and satisfying the hypotheses of Theorem 3.5.

It suffices to show that $\lim D \rightarrow \lim D^{\prime \prime}($ resp. $\lim D$ is an ANR) is a fibration for each proper full subdiagram $D^{\prime \prime}$ of $D$. Choose any initial vertex $a$ of $D$ (that means no arrow ends at $a$ ) not in $D^{\prime \prime}$ and form a new diagram $D^{\prime}$ from $D$ by removing all the arrows starting at $a$. By the inductive assumption $\lim D^{\prime} \rightarrow$ $\lim D(a)$ is a fibration (resp. $\lim D^{\prime}$ and $\lim D(a)$ are ANRs). Consider the natural map $\lim D^{\prime} \rightarrow \lim D(a)$ and apply Lemma 3.6 to the diagram $D^{\prime \prime \prime}$

$$
\begin{gathered}
X(a) \\
\downarrow \\
\lim D(a) \leftarrow \lim D^{\prime} .
\end{gathered}
$$

Thus $\lim D^{\prime \prime \prime} \rightarrow \lim D^{\prime}$ is a fibration (resp. $\lim D^{\prime \prime \prime}$ is an ANR). Consequently $\lim D^{\prime \prime \prime} \rightarrow \lim D^{\prime \prime}$ is a fibration. Since it is clear that $\lim D^{\prime \prime \prime}=\lim D$, the theorem follows. 
3.7. Construction. Given a fibrant diagram $D$ we can form a new diagram $\operatorname{dr}(D)$ (called the derived diagram) whose vertices are full subdiagrams of $D$. The space at the vertex $D^{\prime}$ of $\operatorname{dr}(D)$ is $\lim D^{\prime}$. The arrow from $D^{\prime}$ to $D^{\prime \prime}$ exists only if $D^{\prime \prime}$ is a full subdiagram of $D^{\prime}$, and it represents the map $\lim D^{\prime} \rightarrow$ $\lim D^{\prime \prime}$.

3.8. Proposition. If $D$ is a fibrant diagram, then its derived diagram is also fibrant.

Proof. Any full subdiagram $E$ of $\operatorname{dr}(D)$ gives rise to the minimal full subdiagram $\smile E$ of $D$ which contains all the vertices of $E$. Notice that $\lim E \equiv$ $\lim (\smile E)$ and if $E^{\prime} \subset E$, then $\lim E \rightarrow \lim E^{\prime}$ corresponds to $\lim (\smile E) \rightarrow$ $\lim \left(\smile E^{\prime}\right)$. Proposition 3.8 follows.

3.9. Definition. An inverse system $X=\left\{X_{a}, p_{a a^{\prime}}, A\right\}$ of topological spaces is called fibrant provided for each $b$ in $A$ the diagram $X(b)=\left\{X_{a}, p_{a a^{\prime}}, A^{\prime}(b)\right\}$, where $A^{\prime}(b)=\{a \in A: a \leq b\}$, is fibrant. In particular, $A^{\prime}(b)$ is finite. A space $Z$ is called fibered if it is the inverse limit of a fibrant system of ANRs (this is a generalization of fibered compacta from $[\mathrm{G}]$ ).

\section{StRoNg ShaPe EQUivalenCes IN PRO-TOP}

pro-TOP is the category in which objects are inverse systems in TOP. The set of morphisms (called maps) from $X=\left\{X_{a}, p_{a a^{\prime}}, A\right\}$ to $Y=\left\{Y_{b}, q_{b b^{\prime}}, B\right\}$ is defined by

$$
\operatorname{pro-TOP}(X, Y)=\underset{b}{\lim } \underset{a}{\lim } \operatorname{TOP}\left(X_{a}, Y_{b}\right) \text {. }
$$

In case $B$ is a one-point set

$$
\operatorname{pro-TOP}(X, Y)=\underset{a}{\lim }\left\{\operatorname{TOP}\left(X_{a}, Y\right),\left(p_{a a^{\prime}}\right)^{*}, A\right\} \text {. }
$$

So it is clear how to compose $f: X \rightarrow Y$ and $g: Y \rightarrow Z$ in case $Y$ and $Z$ are topological spaces. In the general case $f: X \rightarrow Y=\left\{Y_{b}, q_{b b^{\prime}}, B\right\}$ is simply a family $\left\{f_{b}: X \rightarrow Y_{b}, b \in B\right\}$ such that $f_{b}=q_{b b^{\prime}} f_{b^{\prime}}$ for $b<b^{\prime}$. If

$$
g=\left\{g_{c}: Y \rightarrow Z_{c}, c \in C\right\}: Y \rightarrow Z=\left\{Z_{c}, r_{c c^{\prime}}, C\right\}
$$

we choose for each $c \in C$ a representative $g_{b(c) c}: Y_{b(c)} \rightarrow Z_{c}$ and put

$$
g f=\left\{g_{b(c) c} \cdot f_{c}: X \rightarrow Z_{c}, c \in C\right\}: X \rightarrow Z \text {. }
$$

The identity map id $X \rightarrow X$ is $\left\{p_{a}: X \rightarrow X_{a}, a \in A\right\}$, where id : $X_{a} \rightarrow X_{a}$ represents $p_{a} . \quad p_{a}$ is called a projection map. Notice that for

$$
f=\left\{f_{b}: X \rightarrow Y_{b}, b \in B\right\}: X \rightarrow Y
$$

we have $f_{b}=p_{b} f$ for all $b \in B$.

If $X=\left\{X_{a}, p_{a a^{\prime}}, A\right\}$ and $Y$ is a topological space, we define $X \times Y$ as $\left\{X_{a} \times Y, p_{a a^{\prime}} \times \mathrm{id}, A\right\}$. In particular we can talk about $X \times I$ and inclusion maps $i_{t}: X \rightarrow X \times I$ for $0 \leq t \leq 1$. Thus $H: X \times I \rightarrow Z$ is a homotopy 
joining $H i_{0}$ and $H i_{1}$. In this way one gets the homotopy category $H$ (pro-TOP) of pro-TOP. The homotopy category HTOP of TOP is a full subcategory of $H$ (pro-TOP) and many notions and terminology will be the same or analogous.

Given a cardinal number $\mathfrak{m} \geq \aleph_{0}$ let $\operatorname{TOP}(\mathfrak{m})$ (resp. ANR $(\mathfrak{m})$ ) be the full subcategory of TOP which objects are spaces (resp. ANRs) of density (= the least cardinality of a dense subset) less than or equal to $\mathfrak{m}$.

A map $f: X \rightarrow Y$ of pro-TOP is a shape-equivalence (resp. m-shape equivalence) if for each $P \in \mathrm{ANR}$ (resp. $P \in \operatorname{ANR}(\mathfrak{m})$ ), the function $f^{*}:[Y, P] \rightarrow$ $[X, P]$ is a bijection between sets of homotopy classes $[Y, P]$ and $[X, P]$.

4.1. Definition. A shape equivalence (resp. m-shape equivalence) $f: X \rightarrow Y$ of pro-TOP is called a strong shape equivalence (resp. strong $m$-shape equivalence) if for any maps $g, h: Y \rightarrow P \in \mathrm{ANR}$ (resp. $P \in \operatorname{ANR}(\mathfrak{m})$ ) and a homotopy $H: X \times I \rightarrow P$ joining $g f$ and $h f, H$ is homotopic rel. $X \times\{0,1\}$ to $H^{\prime} \cdot(f \times$ id $)$ for some homotopy $H^{\prime}: Y \times I \rightarrow P$ joining $g$ and $h$.

As in the case of TOP the homotopy $H^{\prime}$ in the above definition is unique in the sense that any other homotopy $H^{\prime \prime}: Y \times I \rightarrow P$ joining $g$ and $h$ such that $H$ is homotopic rel. $X \times\{0,1\}$ to $H^{\prime \prime} \cdot(f \times$ id $)$ must be homotopic to $H^{\prime}$ rel. $Y \times\{0,1\}$.

4.2. Examples. (a) If each $f_{a}: X_{a} \rightarrow Y_{a^{\prime}}, a \in A$, is a strong shape equivalence, then the level preserving map $f=\left\{f_{a}\right\}_{a \in A}$ is a strong shape equivalence.

(b) $Y=\left\{Y_{a}, p_{a a^{\prime}}, A\right\}$ is the system of all open neighborhoods of a closed subset $X$ of an $M \in$ ANR and each $f_{a}: X \rightarrow Y_{a}$ is the inclusion.

Example (a) is clear. Example (b) follows from the fact that every map $g: A \rightarrow P \in \mathrm{ANR}$, where $A$ is a closed subset of metrizable space $B$, extends onto a neighborhood of $A$ in $B$. Thus every map $g: X \rightarrow P \in$ ANR factors through $Y$. If

$$
H: X \times I \cup U \times\{0,1\} \rightarrow P \in \text { ANR }
$$

is a map, $H$ extends onto $V \times I$ for some neighborhood $V$ of $X$ in $U$. Therefore every homotopy $H: X \times I \rightarrow P \in$ ANR joining two maps $f \mid X$ and $g \mid X$, where $f, g: Y \rightarrow P \in$ ANR factors as $H=H^{\prime} \mid X \times I$ for some homotopy $H^{\prime}: Y \times I \rightarrow P \in$ ANR joining $f$ and $g$.

4.3. Proposition. Suppose $f \cdot X=\left\{X_{a}, p_{a a^{\prime}}, A\right\} \rightarrow Y=\left\{Y_{b}, q_{b b^{\prime}}, B\right\}$ is a strong $\mathrm{m}$-shape equivalence. If the density of each $X_{a}$ and each $Y_{b}$ is at most $\mathfrak{m}$, then $f$ is a strong shape equivalence.

Proof. Suppose $g: X \rightarrow P \in$ ANR is a map. By Theorem 5 in [M-S, p. 38] $g$ factors as $g=r g^{\prime}$, where $g^{\prime}: X \rightarrow P^{\prime}$ and $P^{\prime}$ is an ANR of density $\leq \mathfrak{m}$. Choose $h: Y \rightarrow P^{\prime}$ with $[h f]=\left[g^{\prime}\right]$. Then $[g]=[r h][f]$, so $f^{*}:[Y, P] \rightarrow$ $[X, P]$ is onto.

Suppose $g, h: Y \rightarrow P \in$ ANR are two maps such that $g f$ and $h f$ are homotopic. Choose a homotopy $H: X \times I \rightarrow P$ joining $g f$ and $h f$. Choose representatives $g_{b}: Y_{b} \rightarrow P$ of $g$ and $h_{b}: Y_{b} \rightarrow P$ of $h$. Then choose a 
homotopy $H_{a}: X_{a} \times I \rightarrow P$ joining representatives of $g_{b} f$ and $h_{b} f$. Then $g_{b}\left(Y_{b}\right) \cup h_{b}\left(Y_{b}\right) \cup H_{a}\left(X_{a} \times I\right)$ can be embedded as a closed subset of $P^{\prime} \in \operatorname{ANR}(\mathfrak{m})$ such that

$$
u \mid\left(g_{b}\left(Y_{b}\right) \cup h_{b}\left(Y_{b}\right) \cup H_{a}\left(X_{a} \times I\right)\right)=\mathrm{id}
$$

for some map $u: P^{\prime} \rightarrow P$ (see [M-S, proof of Theorem 5, p. 38]). Now we have maps $g^{\prime}=g_{b} p_{b}: Y \rightarrow P^{\prime}, h^{\prime}=h_{b} p_{b}: Y \rightarrow P^{\prime}$ and a homotopy $H^{\prime}=H p_{a}: X \times I \rightarrow P^{\prime}$ joining $g^{\prime}$ and $h^{\prime}$. Notice that $u g^{\prime}=g, u h^{\prime}=h$ and $u H^{\prime}=H$. Since $H^{\prime}$ is homotopic rel. $X \times\{0,1\}$ to $G(f \times$ id $)$ for some homotopy $G: Y \times I \rightarrow P^{\prime}$ joining $g^{\prime} f$ and $h^{\prime} f$, we conclude that $H$ is homotopic rel. $X \times\{0,1\}$ to $u G(f \times$ id $)$ and $u G: Y \times I \rightarrow P$ is a homotopy joining $g$ and $h$.

4.4. Theorem. If $f: X \rightarrow Y$ is a strong shape equivalence (resp. strong $\mathfrak{m}$ shape equivalence) and $Z \in$ pro-ANR (resp. $Z \in$ pro-ANR(m)) is fibrant then for any $g: X \rightarrow Z$ there is a unique homotopy class $h: Y \rightarrow Z$ with $h[f]=[g]$. Proof. Suppose $Z=\left\{Z_{a}, p_{a a^{\prime}}, A\right\}$ and $g=\left\{g_{a}\right\}: X \rightarrow Z$.

Special case. $f=i$ is an inclusion map and $(Y, X)$ has the homotopy extension property with respect to any topological space. That means $Y=\left\{Y_{b}, q_{b b^{\prime}}\right.$, $B\}, X=\left\{X_{b}, q_{b b^{\prime}}, B\right\}$ and each $X_{b}$ is a closed subset of $Y_{b}$ such that $\left(Y_{b}, X_{b}\right)$ has the homotopy extension property with respect to any space.

Proof of the special case. Since $i$ is a shape equivalence (resp. m-shape equivalence), for each $a \in A$ there is an extension map $k_{a}: Y \rightarrow Z_{a}$ of $g_{a}$. We are going to construct extensions $h_{a}: Y \rightarrow Z_{a}$ of $g_{a}$ such that $p_{a a^{\prime}} h_{a^{\prime}}=h_{a}$ for $a<a^{\prime}$. The construction will be by induction on the number of predecessors $n(a)$ of $a$. In case $n(a)=0$ put $h_{a}=k_{a}$. Suppose $h_{a}$ is defined for all $a$ with $n(a)<m, m \geq 1$. Given $a^{\prime}$ with $n\left(a^{\prime}\right)=m$ the maps $h_{a}, a<a^{\prime}$, define a map $h: Y \rightarrow \lim Z\left(a^{\prime}\right)$. Notice that $\left(Z_{a^{\prime}} \rightarrow \lim Z\left(a^{\prime}\right)\right) g_{a^{\prime}}=h \mid X$. Since

$$
\left(Z_{a^{\prime}} \rightarrow \lim Z\left(a^{\prime}\right)\right) k_{a^{\prime}} \mid X=\left(Z_{a^{\prime}} \rightarrow \lim Z\left(a^{\prime}\right)\right) g_{a^{\prime}}
$$

and $i$ is a strong shape equivalence (resp. strong $\mathrm{m}$-shape equivalence) there is a homotopy $H: Y \times I \rightarrow \lim Z\left(a^{\prime}\right)$ joining $\left(Z_{a^{\prime}} \rightarrow \lim Z\left(a^{\prime}\right)\right) k_{a^{\prime}}$ and $h$ which is stationary on $X \times I$. By [W, Theorem 7.16, p. 35] there is an extension $H^{\prime}: Y \times I \rightarrow Z_{a^{\prime}}$ of $H$ such that $H_{0}^{\prime}=k_{a^{\prime}}$ and $H^{\prime} \mid X \times I$ is stationary. Put $h_{a^{\prime}}=H_{1}^{\prime}$.

Suppose $h, h^{\prime}: Y \rightarrow Z$ are two maps with $h \mid X$ homotopic to $h^{\prime} \mid X$. Notice that $(Y \times I, X \times I \cup Y \times\{0,1\})$ has the homotopy extension property with respect to any space (see [W], p. 22) and that the inclusion $X \times I \cup Y \times\{0,1\} \rightarrow Y \times I$ is a strong shape equivalence (resp. strong $m$-shape equivalence). Using the first part of the proof we can extend the homotopy joining $h \mid X$ and $h^{\prime} \mid X$ to a homotopy joining $h$ and $h^{\prime}$.

General case. Replace $f$ by a level preserving map $f^{\prime}: X^{\prime} \rightarrow Y^{\prime}$ and apply the special case to the inclusion of $X^{\prime}$ into the mapping cylinder of $f^{\prime}$. 
4.5. Corollary. If $f: X \rightarrow Y \in$ pro-ANR (resp. $Y \in$ pro-ANR(m)) and $g$ : $X \rightarrow Z \in$ pro-ANR (resp. $Z \in$ pro-ANR $(\mathfrak{m})$ ) are strong shape equivalences (resp. strong $m$-shape equivalences) and both $Y, Z$ are fibrant then there is a unique homotopy equivalence $h: Y \rightarrow Z$ with $h[f]=[g]$.

4.6. Theorem. There is a functor $R_{\mathrm{m}}$ : pro-TOP $\rightarrow \operatorname{pro}-\mathrm{ANR}(\mathfrak{m})$ and a natural transformation $i_{\mathrm{m}}$ : id $\rightarrow R_{\mathrm{m}}$ from the identity functor to $R_{\mathrm{m}}$ such that the following conditions are satisfied:

(a) $i_{\mathfrak{m}}(X): X \rightarrow R_{\mathrm{m}}(X)$ is a strong $\mathfrak{m}$-shape equivalence for each $X \in$ pro-TOP,

(b) $R_{\mathrm{m}}(X)$ is a fibrant object for each $X$,

(c) $R_{\mathfrak{m}}(f) \approx R_{\mathfrak{m}}(g)$ if $f \approx g$.

Proof. Let $\mathrm{ANR}_{\mathrm{m}}$ be the set of all ANRs contained in $I^{\mathrm{m}}$. Then any ANR of density at most $\mathfrak{m}$ is homeomorphic to one of the elements of $\mathrm{ANR}_{\mathfrak{m}}$. Let $D_{\mathrm{m}}$ be the set of all fibrant diagrams in $\mathrm{ANR}_{\mathrm{m}}$. Given $X$ in TOP let $A(X)$ be the set of all pairs $\left(f_{D}, D\right)$ such that $D$ is a fibrant diagram in $\operatorname{ANR}_{\mathrm{m}}$ and $f_{D}: X \rightarrow \lim D$. We put $\left(f_{D}, D\right)<\left(f_{D^{\prime}}, D^{\prime}\right)$ if $D$ is a proper full subdiagram of $D^{\prime}$ and $f_{D}=\left(\lim D^{\prime} \rightarrow \lim D\right) f_{D^{\prime}}$. This turns $A(X)$ into a directed set such that each element has only finitely many predecessors. Indeed, given $\left(f_{D}, D\right)$ and $\left(f_{D^{\prime}}, D^{\prime}\right)$ one can form a new diagram $D^{\prime \prime}$ which is the disjoint union of $D$ and $D^{\prime}$. Then $\lim D^{\prime \prime}=(\lim D) \times\left(\lim D^{\prime}\right)$ so we put $f_{D^{\prime \prime}}=f_{D} \times f_{D^{\prime}}$. Then $\left(f_{D}, D\right)<\left(f_{D^{\prime \prime}}, D^{\prime \prime}\right)$ and $\left(f_{D^{\prime}}, D^{\prime}\right)<\left(f_{D^{\prime \prime}}, D^{\prime \prime}\right)$.

Put $R_{\mathfrak{m}}(X)=\left\{X_{a}, p_{a a^{\prime}}, A(X)\right\}$, where for $a=\left(f_{D}, D\right)$ we put $X_{a}=\lim D$ and $p_{a a^{\prime}}=\left(\lim D^{\prime} \rightarrow \lim D\right)$ for $a^{\prime}=\left(f_{D^{\prime}}, D^{\prime}\right)>\left(f_{D}, D\right)$. The map $i_{\mathrm{m}}(X)$ : $X \rightarrow R_{\mathfrak{m}}(X)$ is defined as $i_{\mathfrak{m}}(X)_{a}=f_{D}$ for $a=\left(f_{D}, D\right)$.

Given a morphism $g: X \rightarrow Y$ of TOP define $R_{\mathrm{m}}(g): R_{\mathrm{m}}(X) \rightarrow R_{\mathrm{m}}(Y)$ by putting $\varphi\left(f_{D}, D\right)=\left(f_{D} \cdot g, D\right)$ for $\left(f_{D}, D\right) \in A(Y)$ (this defines an orderpreserving function $\varphi: A(Y) \rightarrow A(X))$ and then defining $R_{\mathrm{m}}(g)_{a}: X_{\varphi(a)} \rightarrow Y_{a}$ (here $\left.a=\left(f_{D}, D\right) \in A(Y)\right)$ as the identity map. It is easy to check that one obtains a functor that way and $R_{\mathrm{m}}(g) \cdot i_{\mathrm{m}}(Y)=i_{m}(Y) \cdot g$. Thus $i_{\mathrm{m}}:$ id $\rightarrow R_{\mathrm{m}}$ is a natural transformation of functors.

Suppose $g: X \rightarrow P \in \operatorname{ANR}(\mathfrak{m})$ is a map. Choose a homeomorphism $h$ : $P \rightarrow P^{\prime} \in \mathrm{ANR}_{\mathrm{m}}$. Then $g$ factors as $h^{-1} \cdot i(X)_{a}$, where $a=(h g, D)$ and $D$ consists of single space $P^{\prime}$. Thus

$$
\operatorname{pro-TOP}\left(R_{\mathfrak{m}}(X), P\right) \rightarrow \operatorname{TOP}(X, P)
$$

is onto for all $P \in \operatorname{ANR}(\mathfrak{m})$. Suppose $g, h: X_{a} \rightarrow P \in \operatorname{ANR}(\mathfrak{m})$ are two maps, $a=\left(f_{D}, D\right)$, such that $g f_{D}$ and $h f_{D}$ are homotopic. Choose a homotopy $H: X \times I \rightarrow P$ joining $g f_{D}$ and $h f_{D}$. Our goal is to find $\left(f_{D^{\prime}}, D^{\prime}\right)>\left(f_{D}, D\right)$ and a homotopy $H^{\prime \prime}: \lim D^{\prime} \times I \rightarrow P$ joining

$$
g\left(\lim D^{\prime} \rightarrow \lim D\right) \text { and } h\left(\lim D^{\prime} \rightarrow \lim D\right)
$$

such that $H=H^{\prime \prime}\left(f_{D^{\prime}} \times\right.$ id $)$. Since $P$ is homeomorphic to an element of $\mathrm{ANR}_{\mathrm{m}}$ 
we may as well assume $P \in \mathrm{ANR}_{\mathrm{m}}$. Consider the diagram $D^{\prime \prime}$

$$
\begin{array}{r}
P^{I} \\
\downarrow e \\
\lim D \stackrel{g \Delta h}{\longrightarrow} P \times P
\end{array}
$$

where $e(\omega)=(\omega(0), \omega(1))$ and $(g \Delta h)(x)=(g(x), h(x))$. Since $e$ is a fibration (see [Sp, Corollary 3, p. 98]), the projection $\lim D^{\prime \prime} \rightarrow \lim D$ is a fibration of ANRs. Observe that the homotopy $H$ can be thought of as a map $H: X \rightarrow P^{I}$ and the diagram

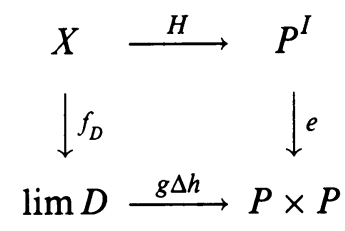

is commutative. Therefore one has maps $f_{D^{\prime \prime}}: X \rightarrow \lim D^{\prime \prime}$ and $H^{\prime \prime}: \lim D^{\prime \prime} \rightarrow$ $P_{I}$ with $H=H^{\prime \prime} f_{D^{\prime \prime}}$ and

$$
f_{D}=\left(\lim D^{\prime \prime} \rightarrow \lim D\right) f_{D^{\prime \prime}}, \quad e H^{\prime \prime}=(g \times h)\left(\lim D^{\prime \prime} \rightarrow \lim D\right) .
$$

Let $D^{\prime}$ be the diagram (strictly speaking we should replace both $\lim D$ and $\lim D^{\prime \prime}$ by equivalent objects of $\mathrm{ANR}_{\mathfrak{m}}$ ) $\lim D^{\prime \prime} \rightarrow \lim D \rightarrow D$ where the last arrow represents all the maps from $\lim D$ to vertices of $D$. Then $\lim D^{\prime}$ is equal to $\lim D^{\prime \prime}$, so we can put $f_{D^{\prime}}=f_{D^{\prime \prime}}$. Now, $H^{\prime \prime}$ is a homotopy joining

$$
g\left(\lim D^{\prime} \rightarrow \lim D\right) \text { and } h\left(\lim D^{\prime} \rightarrow \lim D\right)
$$

such that $H=H^{\prime \prime}\left(f_{D^{\prime}} \times \mathrm{id}\right)$.

The fact that $R_{\mathfrak{m}}(f) \approx R_{\mathfrak{m}}(g)$ if $f \approx g$ is a simple consequence of Theorem 4.4.

For $Y=\left\{Y_{a}, a_{a a^{\prime}}, A\right\}$ define $R_{\mathrm{m}}(Y)$ as

$$
\varliminf_{\mathfrak{m}}\left\{R_{\mathrm{m}}\left(Y_{a}\right), R_{\mathfrak{m}}\left(q_{a a^{\prime}}\right), A\right\}
$$

(recall that pro-TOP is a category with inverse limits). Equivalently, one can define $A(Y)$ as the set of all triples $\left(a, f_{D}, D\right)$ such that $D$ is a fibrant diagram in $\mathrm{ANR}_{\mathrm{m}}$ and $f_{D}: Y_{a} \rightarrow \lim D$. We put $\left(a, f_{D}, D\right)<\left(a^{\prime}, f_{D^{\prime}}, D^{\prime}\right)$ provided $D$ is a proper full subdiagram of $D^{\prime}, a \leq a^{\prime}$ and

$$
\left(\lim D^{\prime} \rightarrow \lim D\right) \cdot\left(f_{D^{\prime}}\right)=f_{D} \cdot q_{a a^{\prime}} .
$$

Similarly one can define $R_{\mathrm{m}}(f)$ for any morphism $f$ of pro-TOP. Using general properties of inverse limits in categories it is easy to complete the proof of Theorem 4.6.

For each topological space $X$ let $d(X)$ be the maximum of $\aleph_{0}$ (the cardinality of the set of integers) and the density of $X$. In case of an inverse system 
$X, d(X)$ is the maximum of densities of elements of the inverse system and of $\aleph_{0}$.

To simplify notation we will denote $R_{d(X)}(X)$ by $R(X)$ and $i_{d(X)}(X)$ by $i(X)$.

The next result shows that the class of fibered spaces plays the same role for strong shape equivalences as the class of ANRs does for shape equivalences. It also shows that using fibered spaces in place of ANRs in the definition of strong shape equivalences does not lead to a new class of maps.

4.7. Theorem. Suppose $f: X \rightarrow Y$ is a map of topological spaces. $f$ is a strong shape equivalence iff for every fibered space $Z$ the function $f^{*}:[Y, Z] \rightarrow$ $[X, Z]$ is a bijection. If $f$ is a strong shape equivalence, then for any maps $g$, $h: Y \rightarrow Z, Z$ fibered, and a homotopy $H: X \times I \rightarrow Z$ joining $g f$ and $h f, H$ is homotopic rel. $X \times\{0,1\}$ to $H^{\prime} \cdot(f \times$ id $)$ for some homotopy $H^{\prime}: Y \times I \rightarrow P$ joining $g$ and $h$.

Proof. Suppose $f$ is a strong shape equivalence and $Z^{\prime}$ is a fibrant object of pro-ANR with $Z=\lim Z^{\prime}$. By Theorem $4.4, f^{*}:\left[Y, Z^{\prime}\right] \rightarrow\left[X, Z^{\prime}\right]$ is a bijection. Since the projection $p: Z=\lim Z^{\prime} \rightarrow Z^{\prime}$ induces a bijection $p^{*}:[A, Z] \rightarrow\left[A, Z^{\prime}\right]$ for each topological space $A$, we get that $f^{*}:[Y, Z] \rightarrow$ $[X, Z]$ is a bijection.

Suppose $f^{*}:[Y, \lim Z] \rightarrow[X, \lim Z]$ is a bijection for each fibrant object $Z$ of pro-ANR. Then $f^{*}:[Y, Z] \rightarrow[X, Z]$ is a bijection. By considering single ANRs we get that $f$ is a shape equivalence. Let $Z=R(X)$. There is $g: Y \rightarrow R(X)$ with $[g f]=[i(X)]$. By Theorem 1.8 (or more precisely, its slight generalization), $f$ is a strong shape equivalence.

Suppose $f: X \rightarrow Y$ is a strong shape equivalence and $H: X \times I \rightarrow Z$ is a homotopy joining $g f$ and $h f, g, h: Y \rightarrow Z, Z$ fibered. Replace $X \times I$ by $D M\left(\mathrm{id}_{X}\right)$. Then $H$ induces a map $G: D M(f) \rightarrow Z$ such that $G|Y \times\{0\}=g, G| Y \times\{1\}=h$. Since $D M(f) \rightarrow M(f) \rightarrow M(f) \times I$ is a strong shape equivalence, by the first part of the proof there is an extension $G^{\prime}: M(f) \times I \rightarrow Z$ of $G$ (recall that $(M(f) \times I, D M(f))$ has the homotopy extension property with respect to any space; see the proof of Theorem 1.3). Using the projection $p: X \times I \times I \rightarrow M(f) \times I$ and a strong deformation retraction of $X \times I \times I$ onto $X \times\{1\} \times I$ one gets that $H$ is homotopic rel. $X \times\{1\} \times\{0,1\}$ to $H^{\prime} \cdot f^{\prime}$ for some homotopy $H^{\prime}: D M\left(\operatorname{id}_{Y}\right) \rightarrow Z$ joining $g$ and $h$.

\section{STRONG SHAPE CATEGORY}

5.1. Definition. The strong shape category $\mathrm{SSh}$ (resp. the strong $\mathrm{m}$-shape category $\mathrm{SSh}_{\mathrm{m}}$ ) is the category whose objects are all topological spaces and the set of morphisms $\operatorname{SSh}(X, Y)$ from $X$ to $Y$ is

$$
H(\text { pro-TOP })(R(X), R(Y))
$$

(resp. $H$ (pro-TOP) $\left.\left(R_{\mathrm{m}}(X), R_{\mathrm{m}}(Y)\right)\right)$ with the obvious composition rule. 
Since the inverse limit functor $\lim :$ pro-TOP $\rightarrow$ TOP induces

$$
\stackrel{\lim }{\longleftarrow} \mathrm{H}(\text { pro-TOP }) \rightarrow \text { HTOP }
$$

one has the functor $r: \mathrm{SSh} \rightarrow$ HTOP (resp. $r_{\mathrm{m}}: \mathrm{SSh}_{\mathrm{m}} \rightarrow$ HTOP). Thus, $r(X)=\lim ^{\prime} R(X)$. Let $p_{X}: r(X) \rightarrow R(X)$ (resp. $\left.p_{\mathrm{m}_{X}}: r_{\mathrm{m}}(X) \rightarrow R_{\mathrm{m}}(X)\right)$ be the natural map from the inverse limit to $R(X)$. Put

$$
i_{X}=\lim _{(}(i(X)): X \rightarrow r(X)
$$

(resp. $\left.\quad i_{\mathrm{m} X}=\lim _{(}\left(i_{\mathrm{m}}(X)\right): X \rightarrow r_{\mathrm{m}}(X)\right)$. In view of Theorems 4.4 and 4.6 there is a functor $S:$ HTOP $\rightarrow \mathrm{SSh}$ (resp. $S_{\mathrm{m}}:$ HTOP $\rightarrow \mathrm{SSh}_{\mathrm{m}}$ ) such that for each map $f: X \rightarrow Y, S[f]: R(X) \rightarrow R(Y)$ (resp. $S_{\mathrm{m}}[f]: R_{\mathrm{m}}(X) \rightarrow R_{\mathrm{m}}(Y)$ ) is the unique morphism of $\mathrm{H}$ (pro-TOP) satisfying $[i(Y) f]=S[f][i(X)]$ (resp. $\left.\left[i_{\mathfrak{m}}(Y) f\right]=S_{\mathrm{m}}[f]\left[i_{\mathfrak{m}}(X)\right]\right)$. Notice that $S[f]$ is an isomorphism iff $f$ is a strong shape equivalence (use results of $\S 4$ ).

5.2. Proposition. If $f: X \rightarrow Y$ is a map then the following diagram is commutative:

$$
\begin{aligned}
& X \stackrel{\left[i_{X}\right]}{\longrightarrow} r(X) \stackrel{\left[p_{X}\right]}{\longrightarrow} R_{d(X)}(X) \\
& \downarrow[f] \quad \downarrow r S[f] \quad \downarrow S[f] \\
& Y \stackrel{\left[i_{Y}\right]}{\longrightarrow} r(Y) \stackrel{\left[p_{X}\right]}{\longrightarrow} R_{d(Y)}(Y) .
\end{aligned}
$$

Thus $i$ is a natural transformation from the identity functor id: HTOP $\rightarrow$ HTOP to the composition $r S$.

Proof. Let $[g]=S[f]$. Since $[i(Y) f]=S[f][i(X)], i(Y) f$ is homotopic to $g i(X)$. Applying the inverse limit functor one gets $r(S[f]) \cdot\left[i_{X}\right]=\left[i_{Y} \cdot f\right]$. The commutativity of the second part of the diagram is a simple consequence of general properties of inverse limits.

5.3. Proposition. The function $\Theta:[X, r(Y)] \rightarrow \operatorname{SSh}(X, Y)$ defined by

$$
\Theta[f]\left[p_{X} i_{X}\right]=\left[p_{Y} f\right],
$$

is a bijection. In particular, $\Theta^{-1}(S[a])=\left[i_{Y} a\right]$ for $a: X \rightarrow Y$.

Proof. $\Theta$ is an injection because $\left[p_{Y} f\right]=\left[p_{Y} g\right]$ implies $[f]=[g]$ in $\mathrm{H}$ (pro-TOP). It is onto since every morphism $X \rightarrow R(Y)$ of pro-TOP factors through $r(Y) \cdot \Theta^{-1}(S[a])=\left[i_{Y} a\right]$ follows from $S[a]\left[p_{X} i_{X}\right]=\left[p_{Y} i_{Y} a\right]$.

5.4. Proposition. $S\left[i_{X}\right]: X \rightarrow r(X)=X^{\prime}$ has a left inverse $g$ in SSh such that $g\left[p_{X^{\prime}} i_{X^{\prime}}\right]=\left[p_{X}\right]$.

Proof. Notice that $p_{X} i_{X}=i(X)$. Take a morphism $g: X^{\prime} \rightarrow X$ of SSh such that $g\left[i\left(X^{\prime}\right)\right]=\left[p_{X}\right]$ (see Theorem 4.4). Then

$$
g S\left[i_{X}\right][i(X)]=g\left[i\left(X^{\prime}\right) i_{X}\right]=\left[p_{X} i_{X}\right]=[i(X)],
$$

so $g S\left[i_{X}\right]=\mathrm{id}_{X}$ in SSh. 
5.5. Corollary. $i_{r(X)}: r(X) \rightarrow r(r(X))$ has a left homotopy inverse.

Proof. Let $g: r(X) \rightarrow X$ be the morphism of SSh such that $g[i(r(X))]=\left[p_{X}\right]$. Apply $\lim _{\leftarrow}$ to get $r(g)\left[i_{r(X)}\right]=\left[\mathrm{id}_{r(X)}\right]$.

5.6. Theorem. Let $X$ be a topological space $X$. The following conditions are equivalent:

(a) $i_{r(X)}: r(X) \rightarrow r(r(X))$ a homotopy equivalence,

(b) $i_{X}: X \rightarrow r(X)$ a strong shape equivalence,

(c) $i_{X}: X \rightarrow r(X)$ a shape equivalence,

(d) $S: \operatorname{HTOP}(Y, r(X)) \rightarrow \operatorname{SSh}(Y, r(X))$ is a bijection for all $Y$, $Y$,

(e) the function $r: \operatorname{SSh}(X, Y) \rightarrow \operatorname{HTOP}(r(X), r(Y))$ is a bijection for all

(f) each strong shape morphism $p: Y \rightarrow X$ factors as $(S[g])^{-1} S[h]$ for some map $h: Y \rightarrow Z$ and some strong shape equivalence $g: X \rightarrow Z$,

(g) $X$ is isomorphic in SSh to $X^{\prime}$ such that

$$
S: \operatorname{HTOP}\left(Y, X^{\prime}\right) \rightarrow \operatorname{SSh}\left(Y, X^{\prime}\right)
$$

is a bijection for all $Y$.

Proof. (a) $\Leftrightarrow$ (b) Using Proposition 5.2 one has $r S\left[i_{X}\right]\left[i_{X}\right]=\left[i_{r(X)}\right]\left[i_{X}\right]$. If $i_{X}$ is a strong shape equivalence, then $r S\left[i_{X}\right]=\left[i_{r(X)}\right]$ by Theorem 4.7. However, $S\left[i_{X}\right]$ is an isomorphism of $\mathrm{H}$ (pro-TOP), so $r S\left[i_{X}\right]$ is a homotopy equivalence.

Suppose $i_{r(X)}$ is a homotopy equivalence. Put $r(X)=X^{\prime}$. Let $g: R\left(X^{\prime}\right) \rightarrow$ $R(X)$ be the unique morphism of $\mathrm{H}$ (pro-TOP) such that $g\left[p_{X^{\prime}} i_{X^{\prime}}\right]=[p x]$. By Proposition 5.4, $g S\left[i_{X}\right]=\mathrm{id}$. Then by applying $\lim$ to $g\left[P_{X^{\prime}} i_{X^{\prime}}\right]=\left[p_{X}\right]$ and $g S\left[i_{X}\right]=\mathrm{id}$ one gets $r(g) r S\left[i_{X}\right]=\mathrm{id}$ and $r(g)\left[i_{X^{\prime}}\right]=\mathrm{id}$. Thus $r(g)$ is a homotopy equivalence and $r S\left[i_{X}\right]=r(g)^{-1}=\left[i_{X^{\prime}}\right]$. Since for any topological space $Z,[Z, R(X)]=[Z, r(X)]$ and $\left[Z, R\left(X^{\prime}\right)\right]=\left[Z, r\left(X^{\prime}\right)\right]$, we get that

$$
S\left[i_{X}\right]^{*}:[Z, R(X)] \rightarrow\left[Z, R\left(X^{\prime}\right)\right]
$$

is a bijection. Consequently,

$$
S\left[i_{X}\right]^{*}: \operatorname{SSh}(Z, X) \rightarrow \operatorname{SSh}\left(Z, X^{\prime}\right)
$$

is a bijection which means that $i_{X}$ is a strong shape equivalence.

(a) $\Leftrightarrow(d)$. The following diagram

$$
\begin{array}{ccc}
\operatorname{HTOP}(Y, r(X)) & \stackrel{S}{\rightarrow} & \operatorname{SSh}(Y, r(X)) \\
& i_{r(X)^{*}} \searrow & \uparrow \boldsymbol{\Theta} \\
& \operatorname{HTOP}(Y, r(r(X)))
\end{array}
$$

is commutative. Indeed, given $f: Y \rightarrow r(X)$,

$$
\Theta\left[i_{r(X)} f\right]\left[p_{Y} i_{Y}\right]=\left[p_{r(X)} i_{r(X)} f\right] .
$$

Also, $S[f]\left[p_{Y} i_{Y}\right]=\left[p_{r(X)} i_{r(X)} f\right]$, so

$$
S[f]\left[p_{Y} i_{Y}\right]=\Theta\left[i_{r(X)} f\right]\left[p_{Y} i_{Y}\right] .
$$

By Theorems 4.4 and 4.6, $S[f]=\Theta\left[i_{r(X)} f\right]$. 
Since $\Theta$ is a bijection (Proposition 5.3), $S$ is a bijection iff $i_{r(X)^{*}}$ is one which happens iff $i_{r(X)}$ is a homotopy equivalence.

(b) $\leftrightarrow$ (c). If $i_{X}$ is a shape equivalence, then $p_{X}$ is a shape equivalence and since $p_{X} i_{X}$ is a strong shape equivalence, by Theorem $1.8, i_{X}$ is a strong shape equivalence.

(b) $\Leftrightarrow(\mathrm{e})$. The following diagram

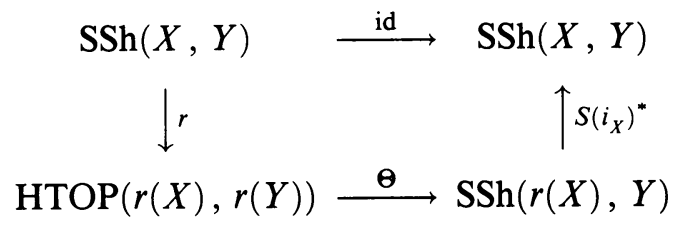

is commutative. Indeed, suppose $g: R(X) \rightarrow R(Y)$. Then $g p_{X}$ factors as $g p_{X}=p_{Y} r(g)$. Also,

$$
\Theta[r(g)]\left[p_{r(X)} i_{r(X)}\right]=\left[p_{Y} r(g)\right]
$$

and

$$
\Theta[r(g)] S\left[i_{X}\right]\left[p_{X} i_{X}\right]=\Theta[r(g)]\left[p_{r(X)} i_{r(X)}\right]\left[i_{X}\right]=\left[p_{Y} r(g)\right]\left[i_{X}\right]=\left[g p_{X}\right]\left[i_{X}\right] .
$$

Thus $\Theta[r(g)] S\left[i_{X}\right]=[g]$.

Consequently

$$
r: \mathrm{SSh}(X, Y) \rightarrow \operatorname{HTOP}(r(X), r(Y))
$$

is a bijection iff $S\left[i_{X}\right]^{*}$ is a bijection which is equivalent to saying that $i_{X}$ is a strong shape equivalence.

(b) $\mapsto\left(\right.$ f) Take a map $h: Y \rightarrow r(X)$ such that $S\left[i_{X}\right] p=S[h]$. Thus $Z=r(X)$ and $g=i_{X}$ works.

(f) $\mapsto$ (b) Consider the morphism $p: Y=r(X) \rightarrow X$ of SSh such that $p\left[p_{Y} i_{Y}\right]=\left[p_{X}\right]$ (see Proposition 5.4) in H(pro-TOP). Let $p=S[g]^{-1} S[h]$, where $g: X \rightarrow Z$ is a strong shape equivalence. Thus $S[g] p=S[h]$. Now,

$$
S[g]\left[p_{X}\right]=S[g] p\left[p_{Y} i_{Y}\right]=S[h]\left[p_{Y} i_{Y}\right]=\left[p_{Z} i_{Z} h\right] .
$$

Applying $\lim _{\longleftarrow}$ we get $r S[g]=\left[i_{Z} h\right]$. Since $r S[g]$ is a homotopy equivalence and $i_{r(Z)}$ has a left homotopy inverse (Proposition 5.4) $i_{Z}$ is a homotopy equivalence. Thus $p$ is an isomorphism and $i_{X}$ is a strong shape equivalence.

(b) $\mapsto(\mathrm{g})$ Obvious.

(g) $\mapsto(\mathrm{f})$ Take a strong shape equivalence $g: X \rightarrow X^{\prime}$. If $p: Y \rightarrow X$ is a morphism of SSh, then there is a map $h: Y \rightarrow X^{\prime}$ such that $S[g] p=S[h]$.

5.7. Problem. Characterize spaces $X$ for which $i_{X}: X \rightarrow r(X)$ is a shape equivalence.

5.8. Remark. We do not know of any space for which $i_{X}: X \rightarrow r(X)$ is not a shape equivalence. It is easy to show that if a compact metric space $X$ is the inverse limit of a sequence of fibrations of ANRs (i.e., $X$ is fibered, see [G]), then $i_{X}: X \rightarrow r(X)$ is a homotopy equivalence. In view of Theorem 5.6, Problem 5.7 is closely related to a problem of Ferry $\left[F_{1}\right]$ of improving 
compacta (see also the example in [Z]). In case each $i_{X}$ is a shape equivalence one would get that $\mathrm{SSh}=(\mathrm{SSE})^{-1}$ HTOP (i.e., $\mathrm{SSh}$ is obtained by localizing HTOP at strong shape equivalences).

Let FIBERED be the full subcategory of TOP whose objects are inverse limits of fibrant objects of pro-ANR. Let HFIBERED be the homotopy category of FIBERED. If $f: X \rightarrow Y$ is a map of topological spaces one has a natural transformation $f^{*}:[Y, \cdot] \rightarrow[X, \cdot]$ of two functors from HFIBERED to SETS; $f^{*}([g])=[f g]$.

5.9. Theorem. The strong shape category SSh is isomorphic to the category whose objects are all topological spaces and morphisms from $X$ to $Y$ are natural transformations $\Pi:[Y, \cdot] \rightarrow[X, \cdot]$ of functors defined on HFIBERED. The transformation corresponding to $S[f]$ equals $f^{*}$.

Proof. Given a natural transformation $\Pi:[Y, \cdot] \rightarrow[X, \cdot]$ we define a morphism $g_{\Pi}: R(X) \rightarrow R(Y)$ of $\mathrm{H}$ (pro-TOP) as follows:

Since $\left[i_{Y}\right]: Y \rightarrow r(Y), \Pi\left[i_{Y}\right]: X \rightarrow r(Y)$ and there is a unique $g_{\Pi}: R(X) \rightarrow$ $R(Y)$ satisfying $g_{\Pi}\left[p_{X} i_{X}\right]=p_{Y} \Pi\left[i_{Y}\right]$ (see Theorems 4.4 and 4.6). Suppose $h: Y \rightarrow r(Z)=\lim _{\longleftarrow} Z$, where $Z$ is a fibrant object of pro-ANR. Let $p_{Z}:$ $r(Z) \rightarrow Z$ be the projection. By Theorems 4.5 and 4.7 there is $g: R(Y) \rightarrow Z$ with $\left[p_{Z} h\right]=g\left[p_{Y} i_{Y}\right]$. Applying inverse limit we get $\left.[h]=r(g)\right]\left[i_{Y}\right]$. Since $\Pi$ is natural, $\Pi[h]=r(g) \Pi\left[i_{Y}\right]$. Thus $g_{\Pi}=g_{\Pi^{\prime}}$ implies $\Pi=\Pi^{\prime}$. Given a strong shape morphism $f: X \rightarrow Y$ we define $\Pi\left[i_{Y}\right]$ as $\Theta^{-1}(f)$ and then $\Pi[h]$ as $r(g) \Pi\left[i_{Y}\right]$, where $g: R(Y) \rightarrow Z$ satisfies $\left[p_{Z} h\right]=g\left[p_{Y} i_{Y}\right]$ (hence $\left.[h]=r(g)\left[i_{Y}\right]\right)$. This establishes a bijection between all natural transformations and strong shape morphisms. Also, it is clear that under the map $\Pi \rightarrow g_{\Pi}$ the composition rule is preserved. If $f=S[a]$, then $\Theta^{-1}(f)=\left[i_{Y} a\right]$ (see Proposition 5.3) and $\Pi[h]=r(g) \Pi\left[i_{Y}\right]=r(g)\left[i_{Y} a\right]=[h a]$.

Let $(\operatorname{ANR}(\mathfrak{m})$, fibrations) be the subcategory of ANR $(\mathfrak{m})$ with the same objects and whose morphisms are Hurewicz fibrations. A strong $m$-shape function $\varphi: X \rightarrow Y$ is a natural transformation from the functor

$$
\operatorname{TOP}(Y, \cdot):(\operatorname{ANR}(\mathfrak{m}), \text { fibrations }) \rightarrow \operatorname{SETS}
$$

to

$$
\operatorname{TOP}(X, \cdot):(\operatorname{ANR}(\mathfrak{m}), \text { fibrations }) \rightarrow \operatorname{SETS} .
$$

Thus for each $P \in \operatorname{ANR}(\mathfrak{m})$ there is a function $\varphi_{P}: \operatorname{TOP}(Y, P) \rightarrow \operatorname{TOP}(X, P)$ such that for every fibration $p: E \rightarrow B$ in $\operatorname{ANR}(\mathfrak{m})$ the diagram

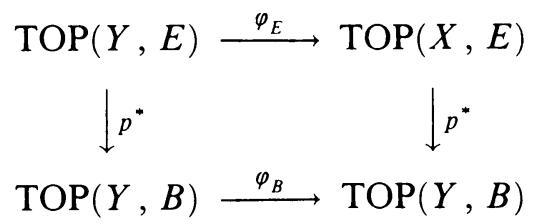

is commutative. Two strong $\mathrm{m}$-shape functions $\varphi$ and $\psi$ are homotopic if there is a strong $\mathrm{m}$-shape function $H: X \times I \rightarrow Y$ with $H \mid X \times\{0\}=\varphi$ 
and $H \mid X \times\{1\}=\psi$. Given a map $f: X \rightarrow Y$ one gets a strong $m$-shape function $f^{*}: \operatorname{TOP}(Y, \cdot) \rightarrow \operatorname{TOP}(X, \cdot) ; f^{*}(g)=f g$. Obviously $f \approx g$ implies $f^{*} \approx g^{*}$. It is clear that topological spaces and homotopy classes of strong $\mathfrak{m}$-shape functions form a category.

5.10. Theorem. The above category is isomorphic to the strong $\mathfrak{m}$-shape category $\mathrm{SSh}_{\mathrm{m}}$. In particular, the homotopy class corresponding to $S_{\mathrm{m}}[f]$ is $\left[f^{*}\right]$.

Proof. Given a strong m-shape function $\varphi: X \rightarrow Y$ define $g(\varphi): X \rightarrow R_{\mathrm{m}}(Y)$ by $g(\varphi)_{a}=\varphi\left(f_{D}\right): X \rightarrow Y_{a}$ if $a=\left(f_{D}, D\right)$. By the naturality of $\varphi$ it is clear that $\tilde{\sigma}(\psi)$ is a map of pro-TOP. Also $g(\varphi)$ is homotopic to $g(\psi)$ if $\varphi$ is homotopic to $\psi$. By Theorems 4.4 and 4.6, $g(\varphi)$ determines uniquely a morphism $f(\varphi): R_{\mathrm{m}}(X) \rightarrow R_{\mathrm{m}}(Y)$ of $\mathrm{H}$ (pro-TOP). Given a morphism $f: R_{\mathrm{m}}(X) \rightarrow R_{\mathrm{m}}(Y)$ of pro-TOP consider $f i_{\mathrm{m}}(X): X \rightarrow R_{\mathrm{m}}(Y)$. Define a strong $\mathrm{m}$-shape function $\varphi(f): X \rightarrow Y$ as follows: Given $P \in \operatorname{ANR}(\mathfrak{m})$ and a map $g: Y \rightarrow P$ choose a homeomorphism $h: P \rightarrow h(P) \in \mathrm{ANR}_{\mathrm{m}}$. Now $a=(h g, h(P))$ is an element of $R_{\mathrm{m}}(Y)$ (here the diagram consists of one space $h(P)$ only). Put $\varphi(f)_{P}(g)=h^{-1} f i_{\mathfrak{m}}(X)_{a}: X \rightarrow P$. If $p: E \rightarrow B$ is a fibration in $\mathrm{ANR}_{\mathrm{m}}$ and $g: Y \rightarrow E$, then $b=(g, E \rightarrow B)$ is an element of $R_{\mathfrak{m}}(Y)$ (here the diagram consists of two spaces $E$ and $B)$. Since $b>a=(g, E)$ and $b>c=(p g, B)$, w have $f i_{\mathfrak{m}}(X)_{a}=f i_{\mathfrak{m}}(X)_{b}$ and $f i_{\mathfrak{m}}(X)_{c}=p f i_{\mathfrak{m}}(X)_{b}$. Consequently, $p \varphi(f)_{E}(g)=\varphi(f)_{B}(p g)$, i.e. $\varphi$ is natural. It is easy to check that $f \approx g$ implies $\varphi(f) \approx \varphi(g)$.

5.11. Remarks. In [B] F. Bauer seems to define a category (with all topological spaces as objects) whose homotopy category is the strong shape category when restricted to compact metrizable spaces. His construction involves both maps and homotopies. We could not find [B, p. 34] any statement that the family of morphisms is a set. For similar reasons we do not know if Theorem 5.10 works for the strong shape category. In particular we do not know if the family of all strong shape functions from $X$ to $Y$ forms a set. The reason it is a set in case of strong $\mathfrak{m}$-shape functions is because $\mathrm{ANR}_{\mathfrak{m}}$ is a set.

The remaining part of this section is devoted to some applications of strong shape theory to function spaces. Function spaces $P^{X}$ (with compact-open topology) have good properties for compactly generated spaces only (see [W, pp. 17$21])$. In this paper we deal with the singular complex of $P^{X}$, so we adjust its definition to work well in the category of all spaces. Let $\mathscr{H}$ lom $(X, P)$ (see [G-Z, p. 131]) be the simplicial complex whose $n$-simplices are maps from $\Delta_{n} \times X$ to $P$ (equivalently; maps from $X$ to $P^{\Delta_{n}}$ ). If $X$ is compactly generated, these correspond precisely to $n$-simplices of the singular complex of $P^{X}$. Here $\Delta_{n}=\left\{\left(t_{0}, \ldots, t_{n}\right) \mid 0 \leq t_{i} \leq 1, \sum t_{i}=1\right\}$ is the standard $n$-simplex. Recall the maps $\delta_{i}: \Delta_{n-1} \rightarrow \Delta_{n}$ and $\sigma_{i}: \Delta_{n+1} \rightarrow \Delta_{n}$;

$$
\delta_{i}\left(t_{0}, \ldots, t_{n-1}\right)=\left(t_{0}, \ldots, t_{i-1}, 0, t_{i}, \ldots, t_{n-1}\right)
$$

and

$$
\sigma_{i}\left(t_{0}, \ldots, t_{n+1}\right)=\left(t_{0}, \ldots, t_{i-1}, t_{i}+t_{i+1}, t_{i+2}, \ldots, t_{n+1}\right) .
$$


The face $\partial_{i}$ and degeneracy $s_{i}$ operators on simplices in $\mathscr{H}(X, P)$ are defined as follows (see [May, p. 2]): $\partial_{i} f=f \cdot\left(\delta_{i} \times\right.$ id) and $s_{i} f=f \cdot\left(\sigma_{i} \times\right.$ id) for any $n$-simplex $f$ of $\mathscr{H} o m(X, P)$. Thus one has for each $P$ a contravariant functor

$$
|\mathscr{H} a m(\cdot, P)|: \text { HTOP } \rightarrow \text { HCW }
$$

from the homotopy category to the homotopy category of CW complexes. stands for the geometric realization functor and

$$
|\mathscr{H} a m([f], P)|:|\mathscr{H} a m(Y, P)| \rightarrow|\mathscr{H} a n(X, P)|,
$$

where $f: X \rightarrow Y$ is a map, is equal to $[|\mathscr{H} a m(f, P)|]$, where $\mathscr{H} a m(f, P)(g)=$ $g($ id $\times f)$ for $g: \Delta_{n} \times Y \rightarrow P$. Our goal is to factor this functor through the strong shape categories.

5.12. Theorem. Suppose $P \in \mathrm{ANR}$ and $\mathfrak{m}$ is a cardinal number greater than or equal to the density of $P$. There is a contravariant functor $\Pi: \mathrm{SSSh}_{\mathrm{m}} \rightarrow \mathrm{HCW}$ such that $\Pi(X)=\mid \mathscr{H}$ om $(X, P) \mid$ and for every map $f: X \rightarrow Y, \Pi\left(S_{\mathrm{m}}[f]\right)=$ $|\operatorname{Lom}([f], P)|$.

Proof. By Theorem 5.10 we can replace $\mathrm{SSh}_{\mathrm{m}}$ by the homotopy category of strong $\mathrm{m}$-shape functions. Suppose $\varphi: X \rightarrow Y$ is a strong $\mathrm{m}$-shape function. We will construct a simplicial map $\alpha_{\varphi}$ from $\mathscr{H} a m(Y, P)$ to $\mathscr{H} a m(X, P)$. We define

$$
\alpha_{\varphi}: \mathscr{H} \operatorname{lom}((Y, P) \rightarrow \mathscr{H} \operatorname{com}(X, P))
$$

as follows: if $f: Y \rightarrow P^{\Delta_{n}}$ is a nondegenerate (i.e., $f \neq s_{i} g$ for any $g$ : $\left.\Delta_{n-1} \times Y \rightarrow P\right) \quad n$-simplex of $\mathscr{H} a m(Y, P), \alpha_{\varphi(f)}=\varphi_{P^{\Delta_{n}}}(f)$. If $f$ is degenerate, $f$ can be expressed uniquely as $f=s_{j_{p}} \cdots s_{j_{1}} g$, where $0 \leq j_{1}<\cdots<j_{p}<n$ and $g$ is a nondegenerate $(n-p)$-simplex of $\mathscr{H} a m(Y, P)$. Put

$$
\alpha_{\varphi}(f)=s_{j_{p}} \cdots s_{j_{1}} \alpha_{\varphi}(g) .
$$

The reason $\alpha_{\varphi}$ is a simplicial map lies in the fact that the map $P^{\Delta_{n}} \rightarrow P^{\Delta_{n-1}}$ given by $f \rightarrow \partial_{i} f$ is a fibration (essentially $\partial_{i} f$ is obtained by restricting $f$ to a face of $\left.\Delta_{n}\right)$. Therefore $\alpha_{\varphi}\left(\partial_{i} f\right)=\partial_{i} \alpha_{\varphi(f)}$. Also $\alpha_{\varphi}\left(s_{i} f\right)=s_{i} \alpha_{\varphi(f)}$, this follows from the way $\alpha_{\varphi}$ was described for degenerate simplices. It is clear that for the composition $\omega$ of $\varphi: X \rightarrow Y$ and $\psi: Y \rightarrow Z$ one has $\alpha_{\omega}=\alpha_{\varphi} \alpha_{\psi}$. If $H: X \times I \rightarrow Y$ is a homotopy between m-shape functions $\varphi$ and $\psi$, then $\varphi=H \cdot i_{0}$ and $\psi=H \cdot i_{1}$, where $i_{0}, i_{1}: X \rightarrow X \times I$ are inclusion induced $m$-shape functions. Thus $\alpha_{\varphi}=\alpha_{i_{0}} \alpha_{H}$ and $\alpha_{\psi}=\alpha_{i_{1}} \alpha_{H}$. Notice that on the level of geometric realizations $\alpha_{i_{0}}$ is homotopic to $\alpha_{i_{1}}$ in the same way as homotopic maps $a, b: A \rightarrow B$ produce homotopic maps $|\mathscr{H} a m(a)|$, $|\mathscr{H} a m(b)|:|\mathscr{H} a m(A)| \rightarrow|\mathscr{H} a m(B)|$ on the level of geometric realizations. Thus (on the level of geometric realizations) $\alpha_{\varphi}$ is homotopic to $\alpha_{\psi}$. This completes the proof of Theorem 5.12. 
5.13. Corollary. Suppose $X$ and $Y$ are nondiscrete shape equivalent compact metrizable spaces. If $P$ is a separable completely metrizable ANR without isolated points, then $P^{X}$ is homeomorphic to $P^{Y}$.

Proof. Since $P^{X}$ is an ANR and $X$ is compact, $\mathscr{H}$ am $(X, P)$ is the singular complex of $P^{X}$ and the natural map $|\mathscr{H} a m(X, P)| \rightarrow P^{X}$ is a homotopy equivalence. By Theorem 5.12, $P^{X}$ is homotopy equivalent to $P^{Y}$ (if $X$ and $Y$ are shape equivalent, then they are isomorphic in SSh-see [D-S $\left.\left.\mathrm{S}_{1}\right]\right)$. Since both $P^{X}$ and $P^{Y}$ are $l_{2}$-manifolds (see [S]), they are homeomorphic (see [B-P, p. 316]).

5.14. Theorem. A map $f: X \rightarrow Y$ is a strong shape equivalence iff for each $P \in$ ANR the map $|\mathscr{H} a m(f, P)|:|\mathscr{H}=m(Y, P)| \rightarrow|\mathscr{H} a m(X, P)|$ is a homotopy equivalence.

Proof. The fact that $|\mathscr{H} a m(f, P)|:|\mathscr{H} a m(Y, P)| \rightarrow|\mathscr{H} a m(X, P)|$ is a homotopy equivalence if $f$ is a strong shape equivalence follows immediately from Theorem 5.12 applied to $\mathfrak{m}=d(P)$. To complete the proof of Theorem 5.14 it suffices to consider the case when $f$ is an inclusion and $(Y, X)$ has the homotopy extension property with respect to any space. Obviously $f$ is a shape equivalence as $[Z, P]$ is the set of path components of $|\mathscr{H a m}(Z, P)|$ for every space $Z$. It is also easy to see that the natural simplicial map $i: \mathscr{H} a m(Y, P) \rightarrow \mathscr{H}$ am $(X, P)$ is a Kan fibration (see [May, p. 25]). Since $i$ is a homotopy equivalence, the fibers of $i$ have trivial homotopy groups. Suppose $H: X \times I \rightarrow P$ is a homotopy joining $g \mid X$ and $h \mid X$ for some maps $g$, $h: Y \rightarrow P$. We may assume (by homotoping $H$ if necessary) that $g|X=h| X$ and $H=(h \mid X) \times$ id. Then both $g$ and $h$ belong to the same fiber of $i$, so there is a 1 -simplex $H^{\prime}: I \times Y \rightarrow P$ of the fiber such that $H^{\prime} \mid\{0\} \times Y=g$ and $H^{\prime} \mid\{1\} \times Y=h$. Since $H^{\prime}$ is an extension of $H$, the proof is completed.

In some cases we do not need to consider all the ANRs $P$ in Theorem 5.14.

5.15. Theorem. Suppose $i: X \rightarrow Y$ is an inclusion of compact metrizable spaces such that the induced map $\left(S^{k}, s\right)^{(Y, x)} \rightarrow\left(S^{k}, s\right)^{(X, x)}$ is a homotopy equivalence. Then $i$ is a strong shape equivalence if one of the following conditions is satisfied:

(a) $Y$ is a subset of the plane and $k \geq 2$,

(b) $\operatorname{dim} Y<k / 2$ and both $X$ and $Y$ are shape 1-connected.

Proof. Since $H_{q}\left(\left(S^{k}, s\right)^{(A, a)}\right)$ is naturally isomorphic to $H^{k-q}(A)$ for $q<$ $2(k-\operatorname{dim} A) \quad$ (reduced groups are considered here-see [Mo]), we infer that $H^{p}(Y) \rightarrow H^{p}(X)$ is an isomorphism for $p \leq 1$ in case (a) and for $p \leq \operatorname{dim} Y$ in case (b). Now, use [Bo, p. 221] (and the proof there), in case (a) and the cohomological version of the Whitehead theorem (see [M-S, p. 155]) plus Theorem 1.13 in this paper. 


\section{STRONG SHAPE FOR METRIZABLE SPACES}

In case of a metrizable space $X$ which is a closed subset of $M \in$ ANR we have a simple description of a strong shape equivalence $i: X \rightarrow\left\{U_{a}, i_{a a^{\prime}}, A\right\}$.

$\left\{U_{a}, i_{a a^{\prime}}, A\right\}$ is the system of all open neighborhoods of $X$ in $M$ and $i_{a}: X \rightarrow U_{a}, i_{a a^{\prime}}: U_{a^{\prime}} \rightarrow U_{a}$ are inclusions.

This calls for equally simple description of the strong shape for metrizable spaces. Let $N(X)$ be the simplicial complex whose vertices are open neighborhoods $U$ of $X$ in $M . s=\left\{U_{0}, \ldots, U_{n}\right\}$ is an $n$-simplex in $N(X)$ iff either $U_{i} \subset U_{j}$ or $U_{j} \subset U_{i}$ for all $i \neq j, i, j \leq n . N(X)$ will be also treated as a directed set: $s<t$ means that $s$ is a proper face of $t$. Given $s=\left\{U_{0}, \ldots, U_{n}\right\}$ we put $U_{s}=\bigcup_{i=1}^{n} U_{i}$ and $\bigcap_{i=1}^{n} U_{i}=V_{s}$. Notice that $V_{s} \subset V_{t}$ if $s>t$, so that one can form the system $\left\{V_{s}, i_{s t}, N(X)\right\}$ such that the inclusion induced map $X \rightarrow\left\{V_{s}, i_{s t}, N(X)\right\}$ is a strong shape equivalence. Now we will find a fibrant object $\left\{U_{s}^{\prime}, r_{s t}, N(X)\right\}$ of pro-ANR and a map

$$
\left\{V_{s}, i_{s t}, N(X)\right\} \rightarrow\left\{U_{s}^{\prime}, r_{s t}, N(X)\right\}
$$

represented by homotopy equivalences $V_{s} \rightarrow U_{s}^{\prime}$ for each $s$. Edwards and Hastings $[\mathrm{E}-\mathrm{H}]$ have a general procedure for replacing objects of pro-TOP by fibrant ones. Cathey and Segal [C-S] showed that in case of ANRs the resulting fibrant object is also in pro-ANR. Using Part III we are able to shorten their proofs significantly.

Let $U_{s}^{\prime}=\left\{a:|s| \rightarrow U_{s}: a(|t|) \subset U_{t}\right.$ for all $\left.t<s\right\}, U_{s}^{\prime}$ inherits the topology from $U_{s}^{|s|}$. Also let $U_{s}^{\prime \prime}=\left\{a:|\partial s| \rightarrow U_{s}: a(|t|) \subset U_{t}\right.$ for all $\left.t<s\right\}$. Given $t<s$ we have the map $r_{t s}: U_{s}^{\prime} \rightarrow U_{t}^{\prime}$ given by $r_{t s}(a)=a|| t \mid$. Notice that in the diagram

$$
U_{s} \rightarrow U_{s} \quad U_{s}^{|s|}{ }^{|\partial s|}
$$

(the horizontal arrow is simply the inclusion) the vertical arrow (= the restriction map) represents a fibration (that is just the homotopy extension theorem) and the limit of that diagram is simply $U_{s}^{\prime}$. Therefore the restriction map $U_{s}^{\prime} \rightarrow U_{s}^{\prime \prime}$ is a fibration and $U_{s}^{\prime}$ is an ANR provided $U_{s}^{\prime \prime}$ is an ANR. Also notice that $U_{s}^{\prime \prime}$ is the limit of the diagram whose vertices are $U_{t}^{\prime}, t<s$ and arrows represent restriction maps $r_{t t^{\prime}}: U_{\left(t^{\prime}\right)}^{\prime} \rightarrow U_{t}^{\prime}$ for $t<t^{\prime}$. So by induction on the dimension of the simplex $s$ one gets that $U_{s}^{\prime}$ is an ANR and $\left\{U_{s}^{\prime}, r_{s t}, N(X)\right\}$ is fibrant. The map $c: V_{s} \rightarrow U_{s}^{\prime}$ is given by $c(u)(x)=u$ for all $x$ in $|s|$. Notice that $c\left(V_{s}\right)$ is a strong deformation retract of $U_{s}^{\prime}$ (homotop a map $a:|s| \rightarrow U_{s}$ in $U_{s}^{\prime}$ to the constant map by linearly deforming $|s|$ to the vertex representing $\left.\bigcap_{i=1}^{n} U_{i}\right)$. Since $c: V_{s} \rightarrow c\left(V_{s}\right)$ is a homeomorphism, $c$ is a homotopy equivalence. Thus $c \mid X: X \rightarrow\left\{U_{s}^{\prime}, r_{s t}, N(X)\right\}$ is a strong shape equivalence and $\left\{U_{s}^{\prime}, r_{s t}, N(X)\right\}$ is equivalent to $R(X)$. 
To understand strong shape morphisms from $X$ to $Y=\operatorname{cl}(Y) \subset M \in$ ANR it is sufficient to analyze maps $f: X \rightarrow\left\{U_{s}^{\prime}, r_{s t}, N(Y)\right\}$ of pro-TOP. Notice that $f_{s}: X \rightarrow U_{s}^{\prime}$ is the same as $f_{s}: X \times|s| \rightarrow U_{s}$ with $f_{s}(X \times|t|) \subset U_{t}$ for all $t<s$. Therefore $f$ corresponds to $f: X \times|N(Y)| \rightarrow M$ with $g(X \times|t|) \subset U_{t}$ for all $t$. Notice that $f \approx g$ iff there is a homotopy $H: X \times I \times|N(Y)| \rightarrow M$ joining $f$ and $g$ such that $H(X \times I \times|t|) \subset U_{t}$ for all $t$. Notice the resemblance of these properties with Quigley's [Q] definition of approaching maps in the compact case.

The composition of

$$
[f]: X \times|N(Y)| \rightarrow M \text { and }[g]: Y \times|N(Z)| \rightarrow P
$$

can be defined as follows:

Treat $f$ as a map from $X$ to $\left\{U_{s}^{\prime}, r_{s t}, N(Y)\right\}$ and $g$ as a map from $Y$ to $\left\{V_{s}^{\prime}, r_{s t}, N(Z)\right\}$. Then $[g]=[G \mid Y]$ for some

$$
G:\left\{U_{s}^{\prime}, r_{s t}, N(Y)\right\} \rightarrow\left\{V_{s}^{\prime}, r_{s t}, N(Z)\right\}
$$

(this is so because $Y \rightarrow\left\{U_{s}^{\prime}, r_{s t}, N(Y)\right\}$ is a strong shape equivalence). Then $[g][f]=[G f]$.

6.1. Theorem. If $f: X \rightarrow Y$ is a strong shape equivalence of metrizable spaces and $Z$ is a locally compact metrizable space, then $f \times \mathrm{id}: X \times Z \rightarrow Y \times Z$ is a strong shape equivalence.

Proof. Choose a locally finite cover $\mathbf{S}$ of $Z$ consisting of compact sets. By Proposition 1.12, the map

$$
f \times \mathrm{id} \mid(f \times \mathrm{id})^{-1}\left(X \times \bigcap A_{i}\right):(f \times \mathrm{id})^{-1}\left(X \times \bigcap A_{i}\right) \rightarrow X \times \bigcap A_{i}
$$

is a strong shape equivalence for each element $A_{1}, \ldots, A_{k}$ of $Y \times \mathbf{S}$. By Theorem 2.9, $f \times$ id is a strong shape equivalence.

6.2. Corollary. If $f: X \rightarrow Y$ and $g: X^{\prime} \rightarrow Y^{\prime}$ are strong shape equivalences of locally compact metrizable spaces, then $f \times g: X \times X^{\prime} \rightarrow Y \times Y^{\prime}$ is a strong shape equivalence.

Given a closed subset $X$ of $M \in$ ANR let $C(N(X))$ be the chain complex of the simplicial complex $N(X)$ and $\Delta(M)$ the singular chain complex of $M$.

6.3. Definition. Suppose $G$ is a group and $k$ is an integer. The strong $k$ homology group ${ }^{s} H_{k}(X ; G)$ of $X$ is the group of all chain homotopy classes of chain maps $\varphi: C(N(X)) \rightarrow \Delta(M) \otimes G$ of degree $k$ satisfying the following condition: For each simplex $s$ of $N(X), \varphi(s) \in \Delta\left(U_{s}\right) \otimes G$. The chain homotopy $D$ between $\varphi$ and $\psi$ must also satisfy $D(s) \in \Delta\left(U_{s}\right) \otimes G$.

Thus for each $n$ we have a homomorphism $\varphi_{n}: C_{n}(N(X)) \rightarrow \Delta_{n+k}(M) \otimes G$ with $\varphi_{n}(s) \in \Delta_{n+k}\left(U_{s}\right) \otimes G$ for $s \in N(X)^{(n)}$. The fact that $\varphi$ is a chain map means that $\partial \cdot \varphi_{n}=\varphi_{n-1} \cdot \partial$ for all $n \geq 0$. 
The above definition was motivated by the idea of changing homotopy to homology. Thus a strong morphism from $\Delta_{k}$ (the standard $k$-simplex) to $X$ is represented by a map $g: \Delta_{k} \times|N(X)| \rightarrow M$ such that $g\left(\Delta_{k} \times|s|\right)$ is contained in $U_{s}$ for each $s$ in $N(X)$. In particular, $g\left|\Delta_{k} \times\right| s \mid$ is a singular $(k+\operatorname{dim}(s))$ chain in $U_{s}$.

6.4. Theorem. ${ }^{s} H_{k}(X ; G)$ is isomorphic to the group $H_{k}^{s}(X ; G)$ introduced by Lisica and Mardešić.

Proof. Since $i: X \rightarrow\left\{U_{a}, i_{a a^{\prime}}, A\right\}$ is a resolution of $X$ in the sense of Mardešic (see [M-S, p. 74]), $H_{k}^{s}(X ; G)$ is the homology group of the chain complex $C_{\#}\left(\left\{U_{a}, i_{a a^{\prime}}, A\right\}\right)$ of strong chains (see $\left.\left[\mathrm{L}^{-\mathrm{M}_{2}}\right]\right)$ of $\left\{U_{a}, i_{a a^{\prime}}, A\right\}$. A strong $k$-chain $(p \geq 0)$ of $\left\{U_{a}, i_{a a^{\prime}}, A\right\}$ is a function $x$ which assigns to every decreasing sequence $a=\left\{U_{0}, \ldots, U_{n}\right\}$ of neighborhoods of $X$ in $M$, a singular $(n+k)$-chain $x_{a}$ of $\Delta\left(U_{0} ; G\right)$. The boundary $d x$ of $x$ is defined as follows:

$$
(-1)^{n}(d x)_{a}=\partial\left(x_{a}\right)-\bigcup_{j=0}^{n}(-1)^{j} x_{a_{j}} .
$$

Here $a_{j}(j \geq 0)$ is the sequence obtained from $a=\left\{U_{0}, \ldots, U_{n}\right\}$ by removing the $j$ th element $U_{j}$. Suppose $x$ is a strong $k$-cycle $(k \geq 1)$; then $a \rightarrow x_{a}$ defines a chain map $x: C(N(X)) \rightarrow \Delta(M) \otimes G$ of degree $k$ such that for each simplex $a$ of $N(X), x_{a} \in \Delta\left(U_{a}\right) \otimes G$. It is also clear that every such chain map arises from a strong $k$-cycle. Suppose $x-y=d z$ and $x, y$ are strong $k$-cycles. We are going to define a chain homotopy $D$ (of degree $k+1$ ) from $x$ to $y$. Put $D(a)=(-1)^{n} z_{a}$ if $a$ is an $n$-simplex of $N(X)$. Then $\partial D(a)+D(\partial a)=x_{a}-y_{a}$. Conversely, chain homotopic maps define strong $k$-chains which difference is a boundary.

6.5. Remark. Originally Lisica and Mardešić $\left[\mathrm{L}-\mathrm{M}_{2}\right]$ introduced strong $k$-chains for $k \geq 0$ only. See [M-P] for a general definition of strong $k$-chains for all integers $k$.

The Lisica-Mardešić strong homology groups have the following interesting property (characteristic for Steenrod homology):

6.6. Theorem. If $X$ is paracompact and $A$ is a closed subset of $X$, then the projection $X \rightarrow X / A$ induces an isomorphism

$$
H_{k}^{s}(X, A ; G) \rightarrow H_{k}^{s}(X / A, p t ; G) .
$$

Proof. Consider the union $X \cup C(A)$ of $X$ and the cone over $A$. By [L-M ${ }_{3}$, p. 32] the inclusion induced homomorphism

$$
H_{k}^{s}\left(X \cup A \times\left[0, \frac{1}{2}\right], A \times\left[0, \frac{1}{2}\right] ; G\right) \rightarrow H_{k}^{s}(X \cup C(A), C(A) ; G)
$$

is an isomorphism (excision property). Since the inclusion induced homomorphism

$$
H_{k}^{s}(X, A ; G) \rightarrow H_{k}^{s}\left(X \cup A \times\left[0, \frac{1}{2}\right], A \times\left[0, \frac{1}{2}\right] ; G\right)
$$


is an isomorphism (the inclusion is a homotopy equivalence), we infer that

$$
H_{k}^{s}(X, A ; G) \rightarrow H_{k}^{s}(X \cup C(A), C(A) ; G)
$$

is an isomorphism. Consider the projection $X \cup C(A) \rightarrow X / A$. By Corollary 2.2 it is a strong shape equivalence. Therefore

$$
\left.H_{k}^{s}(X \cup C(A), C(A) ; G) \rightarrow H_{k}^{s}(X / A), p t ; G\right)
$$

is an isomorphism which completes the proof of Theorem 6.6.

In case $X$ is strongly paracompact Theorem 6.6 was proved by $\mathrm{T}$. Watanabe [Wa]. In full generality it was established by Lisica and Mardešić [L-M $\mathrm{M}_{4}$ ] via a more involved argument than ours.

6.7. Corollary. A shape equivalence $f: X \rightarrow Y$ of paracompact spaces induces an isomorphism $f_{*}: H_{k}^{s}(X ; G) \rightarrow H_{k}^{s}(Y ; G)$ of strong homology groups.

Proof. It suffices to consider the case where $f$ is an inclusion and $X$ is a closed subset of $Y$. By Corollary 2.4, the space $Y / X$ is of trivial shape. By Corollary 1.10 , the inclusion $p t \rightarrow Y / X$ is a strong shape equivalence. Thus $H_{k}^{s}(Y / X ; G)=0$ (reduced groups) and $H_{k}^{s}(X ; G) \rightarrow H_{k}^{s}(Y ; G)$ is an isomorphism by the exactness of strong homology groups.

In the compact metrizable case Corollary 6.7 appears in [C-H].

6.8. Definition. Given an object $X$ of pro-TOP (pointed case) define ${ }^{s} \pi_{k}(X)$ as $\pi_{k}(\lim R(X))$ (this is the group of all homotopy classes of maps from $S_{k}$ to $R(X))$. If $\left\{E_{n}\right\}$ is a spectrum and $X$ a topological space, we have a natural homomorphism

$$
{ }^{s} \pi_{k+m}\left(E_{m} \wedge R(X)\right) \rightarrow{ }^{s} \pi_{k+m+1}\left(E_{m+1} \wedge R(X)\right) .
$$

The direct limit of this sequence we denote by ${ }^{s} E_{k}(X)$.

6.9. Problem. If $\left\{E_{n}\right\}$ is the Eilenberg-Mac Lane spectrum $\{K(G, n)\}$, is ${ }^{s} E_{k}(X)$ isomorphic to the Lisica-Mardešić group $H_{k}^{s}(X ; G)$ ? If $X$ is compact metrizable, is ${ }^{s} E_{k}(X)$ isomorphic to the group introduced by Kahn-KaminkerSchochet [K-K-S]?

\section{REFERENCES}

[B] F. W. Bauer, A shape theory with singular homology, Pacific J. Math. 64 (1976), 25-65.

[Bo] K. Borsuk, Theory of shape, Polish Scientific Publishers, Warsaw, 1975.

[B-P] Cz. Bessaga and A. Pelczynski, Selected topics in infinite-dimensional topology, Polish Scientific Publishers, Warsaw, 1975.

[C] F. Cathey, Strong shape theory, Shape theory and Geometric Topology, Lecture Notes in Math., vol. 870, Springer-Verlag, Berlin and New York, 1981, pp. 215-238.

[C-H] A. Calder and H. M. Hastings, Realizing strong shape equivalences, J. Pure Appl. Algebra 20 (1981), 129-156.

[C-S] F. Cathey and J. Segal, Strong shape theory and resolutions, Topology Appl. 15 (1983), 119-130. 
[D] J. Dugundji, Topology, Allyn and Bacon, 1966.

[D-K] J. Dydak and G. Kozlowski, A generalization of the Vietoris-Begle theorem, Proc. Amer. Math. Soc. 102 (1988), 209-212.

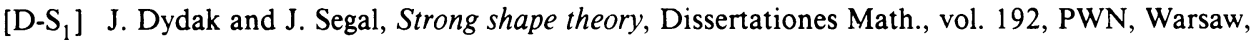
1981, pp. 1-42.

[D-S $\left.\mathrm{S}_{2}\right]$ - Shape theory: An introduction, Lectures Notes in Math., vol. 688, Springer-Verlag, Berlin and New York, 1978, pp. 1-150.

[E] R. Engelking, Outline of general topology, North-Holland, Amsterdam, 1968.

[E-H] D. A. Edwards and H. Hastings, Cech and Steenrod homotopy theories with applications to geometric topology, Lecture Notes in Math., vol. 542, Springer-Verlag, Berlin, 1976.

$\left[\mathrm{F}_{1}\right]$ S. Ferry, A stable converse to the Vietoris-Smale theorem with applications to shape theory, Trans. Amer. Math. Soc. 261 (1980), 369-389.

$\left[\mathrm{F}_{2}\right] \quad$ - Homotopy, simple homotopy, and compacta, Topology 19 (1980), 101-110.

[G] R. Geoghegan, Fibered stable compacta have finite homotopy type, Proc. Amer. Math. Soc.

[G-Z] P. Gabriel and M. Zisman, Calculus of fractions and homotopy theory, Ergeb. Math. Grenzaeb., vol. 35, Springer-Verlag, Berlin and New York, 1967.

[H] S. T. Hu, Theory of retracts, Wayne State Univ. Press, Detroit, 1965.

[K-K-S] D. S. Kahn, J. Kaminker and C. Schochet, Generalized homology theories on compact metric spaces, Michigan Math. J. 24 (1977), 203-224.

[K] G. Kozlowski, Images of ANR's, Mimeographed notes, Univ. of Washington, Seattle, 1974.

$\left[\mathrm{L}-\mathrm{M}_{1}\right]$ Ju. T. Lisica and S. Mardešić, Coherent prohomotopy and strong shape theory, Glas. Mat. 19 (1984), 335-399.

[L-M $\left.\mathrm{M}_{2}\right]$ _ Strong homology of inverse systems of spaces. I, Topology Appl. 19 (1985), 29-43.

[L-M $\left.\mathrm{M}_{3}\right]$ _ Strong homology of inverse systems of spaces. III, Topology Appl. 20 (1985), 29-37.

[L-M $\left.\mathrm{M}_{4}\right]$, Steenrod homology, Proc. Geometric and Algebraic Topology, Banach Center Publ., vol. 18, PWN, Warsaw, 1986, pp. 329-343.

[M-P] S. Mardešić and A. V. Prasolov, Strong homology is not additive, Trans. Amer. Math. Soc. 307 (1988), 725-744.

[M-S] S. Mardešić and J. Segal, Shape theory, North-Holland, Amsterdam, 1982.

[May] J. P. May, Simplicial objects in algebraic topology, Van Nostrand, New York, 1967.

[M] J. Milnor, On the Steenrod homology theory, mimeographic notes, Berkeley, 1960.

[Mo] J. C. Moore, On a theorem of Borsuk, Fund. Math. 43 (1955), 195-201.

[Q] J. B. Quigley, An exact sequence from the nth to $(n-1)$ st fundamental group, Fund. Math. 77 (1973), 195-210.

[S] K. Sakai, The space of cross-sections of a bundle, Proc. Amer. Math. Soc. (to appear).

[Sp] E. Spanier, Algebraic topology, McGraw-Hill, New York, 1966.

[St] A. Strom, The homotopy category is a homotopy category, Arch. Math. (Basel) 23 (1973), 435-441.

[Wa] T. Watanabe, Čech homology, Steenrod homology and strong homology. I, Glas. Mat. 27 (1987), 187-238.

[W] George W. Whitehead, Elements of homotopy theory, Springer-Verlag, Berlin and New York, 1978.

[Z] S. Zdravkovska, An example in shape theory, Proc. Amer. Math. Soc. 83 (1981), 594-596.

Department of Mathematics, University of Tennessee, Knoxville, Tennessee 37996 Poland

Institute of Mathematics, University of Warsaw, PKIN, IXP., 00-901 Warszawa, 